The Astrophysical Journal, 275:773-789, 1983 December 15

(C) 1983. The American Astronomical Society. All rights reserved. Printed in U.S.A.

\title{
GLOBULAR CLUSTER GIANT BRANCHES AND THE METALLICITY SCALE
}

\author{
JAY A. Frogel \\ Cerro Tololo Inter-American Observatory ${ }^{1}$ \\ JUDITH G. COHEN \\ Palomar Observatory, California Institute of Technology \\ AND \\ S. E. PERSSON \\ Mount Wilson and Las Campanas Observatories, Carnegie Institution of Washington \\ Received 1983 February 28; accepted 1983 May 24
}

\begin{abstract}
Using the data base of Frogel, Persson, and Cohen and our earlier work, we derive characteristic parameters that describe the systematic behavior of the giants in each of 33 globular clusters. The globular cluster giant branches form a strictly homologous sequence in the H-R diagram, and their ordering correlates well with the metallicity scale published by Zinn. Nevertheless, when the giant branch parameters are used to define a metallicity ranking scheme, it appears that the Zinn abundance scale systematically underestimates the metallicity of clusters with exceptionally blue horizontal branches.

The observed luminosities of the brightest giant in each cluster agree with the theoretical core helium flash luminosity; the small dispersion of these luminosities implies that at a given metallicity the intrinsic scatter in the absolute magnitude of horizontal-branch stars is less than $0.1 \mathrm{mag}$.

The mean CO index increases as metallicity increases; analysis of the residuals from the mean relationships both of $\mathrm{CO}$ and of horizontal-branch type against metallicity imply that the $\mathrm{CO}$ abundance is unrelated to the second parameter problem. All metal-rich clusters with an adequate sample of stars show a spread in $\mathrm{CO}$ within each cluster comparable to that seen in 47 Tuc. Metal-poor clusters which exhibit a large star to star spread in CO are NGC 362, 2808, and 6656.

The integrated light measurements of $(V-K)_{0}$ and $\mathrm{CO}$ published by Aaronson and colleagues correlate with the cluster parameters determined from measurements of individual stars in a manner that can be predicted from stellar evolutionary calculations for old populations. The integrated light of such old systems, at least from $V$ to $K$, is therefore well understood.
\end{abstract}

Subject headings: clusters: globular - stars: abundances - stars: late-type

\section{INTRODUCTION}

Six years ago we began a program of obtaining infrared observations ( $\mathrm{JHK}$ magnitudes and $\mathrm{CO}$ and $\mathrm{H}_{2} \mathrm{O}$ band indices) of giant stars in globular clusters (Cohen, Frogel, and Persson 1978). Our initial objective was to assemble a library of infrared colors, magnitudes, and molecular band indices for globular cluster stars drawn from the entire range of cluster metal abundance. These data are of importance in the construction of stellar synthesis models and for a proper understanding of differences and similarities between the stellar populations of globular clusters and early-type galaxies (Aaronson et al. 1978; Frogel, Persson, and Cohen 1980). In many cases, such as for clusters associated with

\footnotetext{
${ }^{1}$ CTIO is operated by AURA, Inc. under National Science Foundation contract AST 78-27879.
}

nearby galaxies, only integrated light observations are feasible, and they must be calibrated by studies of nearby clusters for which both integrated light and individual stellar observations are possible. The present paper, the accompanying one (Frogel, Persson, and Cohen 1983), and the series of papers we and our colleagues have published since 1978 accomplishes this objective. We now have infrared stellar data for 35 clusters; the results are published in eight papers: Cohen, Frogel, and Persson (1978, hereafter GC1) (M3, M13, M92); Frogel, Persson, and Cohen (1979, hereafter GC2) (M71); Persson et al. (1980, hereafter GC3) ( $\omega$ Cen); Cohen et al. (1980, hereafter GC4) (Pal 12); Frogel, Persson, and Cohen (1981a, hereafter GC5) (47 Tuc); Da Costa, Frogel, and Cohen (1981, hereafter GC6) (NGC 3201); Cohen and Frogel (1982, hereafter GC7) (NGC 7006); and 26 additional clusters in Frogel, Persson, and Cohen (1983, hereafter GC8). Only $\omega$ Cen 
and Pal 12 will not be considered any further in this paper.

This homogenous body of infrared data is also valuable in a study of globular clusters themselves. Infrared colors and magnitudes of metal-poor cool giants can be used to determine accurate effective temperatures and bolometric luminosities for these stars (GC1, GC5). It is useful to derive from the stellar data a number of parameters which characterize the overall appearance of the giant branch of each cluster in terms of effective temperature, luminosity, color, and molecular band strength. A discussion of these characteristic parameters and their dependence on one another and on independently derived physical parameters is given in $\S$ II-IV.

Section III examines the question of whether our data are consistent in detail with certain basic predictions of the theory of stellar evolution. Specific issues discussed include a comparison of the theoretical and observed location of the core helium flash and of the temperatures of the giant branches. Section IV deals with the overall strength of $\mathrm{CO}$ absorption in cluster stars, its relationship with horizontal branch morphology and cluster metallicity, (i.e., the second parameter problem), and its range within a given cluster.

In $\S \mathrm{V}$ we consider to what extent $V-K$ colors and $\mathrm{CO}$ indices measured from the integrated light of globular clusters are correlated with parameters derived from the individual stellar observations of the same colors and indices. The quality of this correlation is clearly of importance in evaluating the utility of integrated light observations of distant clusters.

The globular cluster metallicity scale is discussed in $\S$ VI, where the characteristic parameters of the cluster giant branches indicate possible problems in the most commonly used abundances. Severe inconsistencies are encountered in dealing with the global giant branch parameters of five clusters; the data for the individual stars in these particular clusters and for two others are considered in an appendix, where possible explanations are suggested in terms of erroneous distance moduli, reddenings, or photometric errors. The final section is a summary of the most important results. Preliminary discussions of parts of this paper have appeared in Frogel (1980) and in Frogel, Persson, and Cohen (1981b).

\section{CLUSTER PARAMETERS}

The clusters treated in this paper are listed in Table 1. Columns (2) and (3) identify the clusters. The next two columns give the color excesses and extinction-corrected distance moduli from the sources noted in GC8. The sixth column contains the metallicity estimates given by Zinn (1980) or, for the two cases in parentheses, from the tabulation of Harris and Racine (1979). Zinn's metallicity scale (henceforth $[\mathrm{Fe} / \mathrm{H}]_{\mathrm{Z}}$ ) has been chosen both because of its extensiveness and its apparently high degree of internal consistency. With few exceptions, the uncertainty for any given $[\mathrm{Fe} / \mathrm{H}]_{Z}$ determination which arises from observational errors alone is, according to Zinn, 0.05-0.20 dex. The seventh column characterizes the morphology of the horizontal branch according to Kukarkin (1974): 0.0 is a predominantly blue one, while 1.0 is a predominantly red one.

Zinn's (1980) metallicity scale has been used to divide the clusters into five groups. Group A clusters have $[\mathrm{Fe} / \mathrm{H}]_{\mathrm{Z}}>-0.7$, while group $\mathrm{B}$ covers the interval $-1.4<[\mathrm{Fe} / \mathrm{H}]_{\mathrm{Z}}<-0.7$; group $\mathrm{C}$ includes clusters with $-1.8<[\mathrm{Fe} / \mathrm{H}]_{\mathrm{Z}} \leq-1.4$, and group $\mathrm{D}$ includes the most metal-poor clusters. To maintain the groups approximately equal in size, group $\mathrm{C}$ was subdivided into group $\mathrm{C} 1$ with $-1.6<[\mathrm{Fe} / \mathrm{H}]_{\mathrm{Z}} \leq-1.4$ and group $\mathrm{C} 2$ which covers the remainder of the interval. The group membership of each cluster is indicated in column (1).

Columns (8)-(15) are based on observations in GC8 and in papers $\mathrm{GC} 1, \mathrm{GC} 2, \mathrm{GC} 5, \mathrm{GC} 6$, and $\mathrm{GC} 7$. Column (8) lists the absolute bolometric luminosity of the brightest star in each cluster where only the long period variables (LPVs) have been excluded. The next four columns tabulate the characteristic parameters which define the overall locations of the GBs of the clusters in color-magnitude diagrams and in the physical H-R diagrams. In order to derive these parameters, smooth curves were first drawn by hand through the ridge line of the GB stars on the appropriate plots. AGB stars identified from optical data alone were ignored in the drawing of the mean GBs. The parameters are determined from the mean GBs for each cluster as follows: $T_{e}(\mathrm{~GB})$ is the effective temperature of the mean $\mathrm{GB}$ at an $M_{\text {bol }}=-3.0 ;(V-K)_{0}(\mathrm{~GB})$ and $(J-K)_{0}(\mathrm{~GB})$ are the colors of the mean GB at $M_{K_{0}}=-5.5$; and $M_{K_{0}}(\mathrm{~GB})$ is determined at $(V-K)_{0}=3.0 . \mathrm{CO}(\mathrm{GB})$ is the median $\mathrm{CO}$ value of the stars in the neighborhood of $(V-K)_{0}$ $=3.0 . \sigma \mathrm{CO}(\mathrm{GB})$ in column (14) is an indicator of the spread in the stellar $\mathrm{CO}$ values in the region of the GB with $(V-K)_{0}=3.0-3.5$. Figure 1 shows how the GB parameters are defined, with the giant branches plotted representing the metallicity groups of Table 1 .

Finally, $[\mathrm{Fe} / \mathrm{H}]_{\mathrm{IR}}$, in column (16), is a new, preliminary metallicity scale described in $\S$ VI which uses the infrared data for relative rankings of the clusters and the spectroscopic work of Cohen $(1982,1983)$ for an absolute calibration.

\section{THE METALLICITY DEPENDENCE OF CLUSTER GIANT BRANCHES IN THE H-R DIAGRAM}

A large body of stellar interior calculations defines detailed theoretical giant branches as a function of metallicity, age, and other parameters. Many previous analyses of observational material (including $\mathrm{GC} 1-\mathrm{GC} 7$ ) for globular cluster giants have demonstrated that qualitative agreement with the theoretical calculations exists. Using our large set of homogenous data we seek 


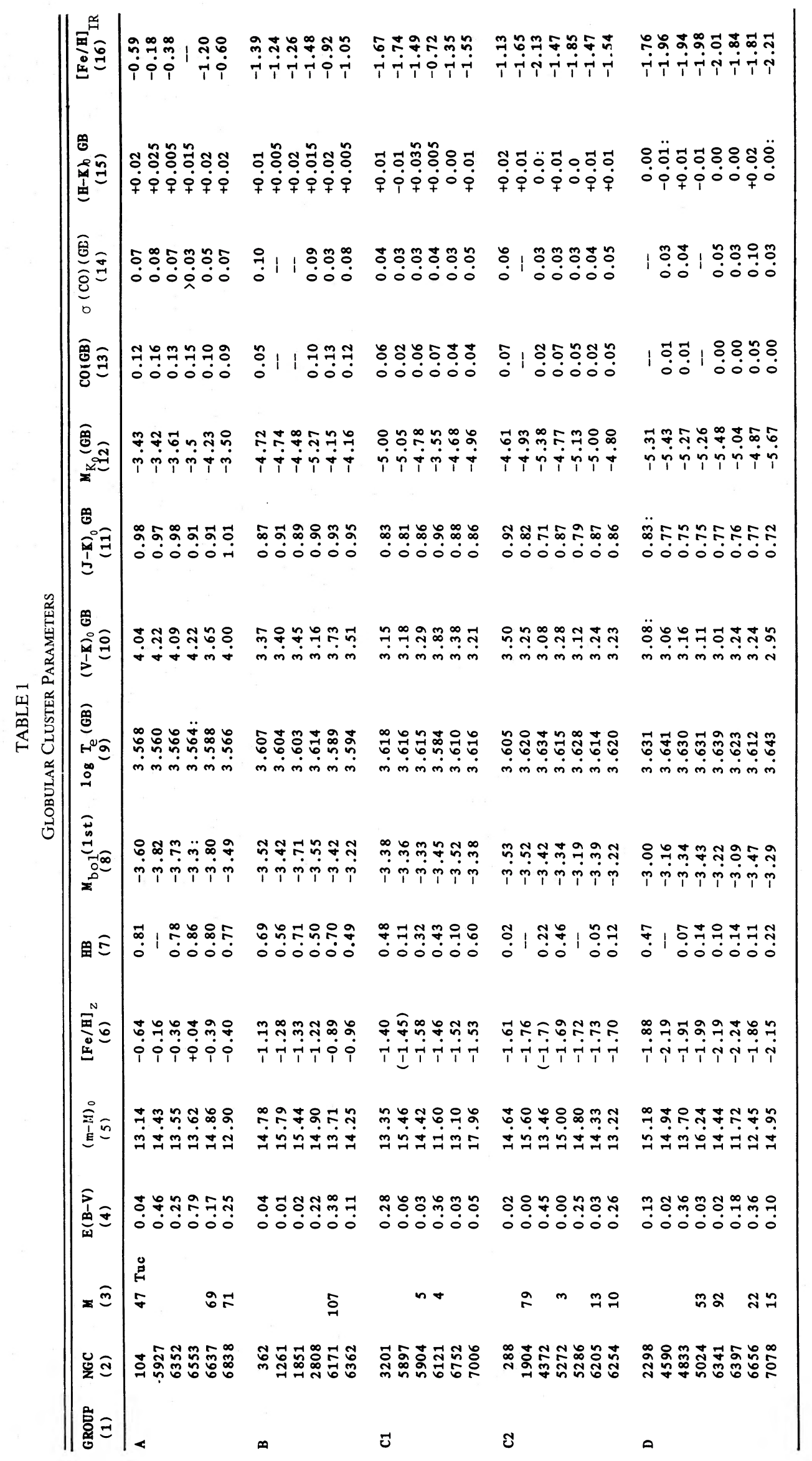

(C) American Astronomical Society - Provided by the NASA Astrophysics Data System 


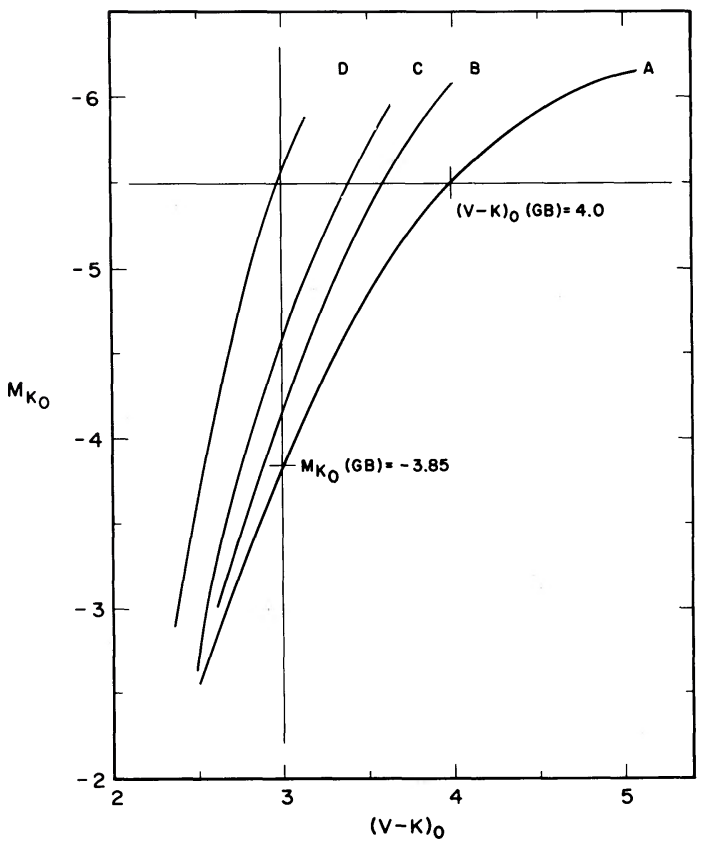

FIG. 1.-A schematic illustration of the definition of the giant branch parameter $M_{K}$, the absolute $K$ magnitude of the mean giant branch at $(V-K)_{0}=3.0$, and the parameter $(V-K)_{0}(\mathrm{~GB})$, the color of the mean $\mathrm{GB}$ at $M_{K_{0}}=5.5$.

to determine whether detailed quantitative agreement exists. In order to carry this out, we have produced physical H-R diagrams ( $T_{\text {eff }}$ versus $\left.M_{\text {bol }}\right)$ from our observational data as described in GC8. A question of critical concern here is the distance scale. As stated in GC8, we have adopted horizontal-branch magnitudes $M_{v_{0}}$ of +0.6 for metal-poor clusters with $[\mathrm{Fe} / \mathrm{H}]_{\mathrm{Z}}<$
$-1.0,+0.8$ for the most metal-rich clusters with $[\mathrm{Fe} / \mathrm{H}]_{\mathrm{Z}}<-0.8$, and +0.7 for the clusters with -1.0 $\leq[\mathrm{Fe} / \mathrm{H}]_{\mathrm{Z}} \leq-0.8$. A recent review of the absolute magnitude determinations of field RR Lyrae stars is given by Manduca et al. (1981). All analyses of observational data via the Baade-Wesselink method show a trend for the metal-rich field RR Lyrae variables to be somewhat fainter than the metal-poor ones, consistent with the theoretical predictions of Christy (1966) and van Albada and Baker (1973), with a mean absolute visual magnitude of +0.6 . An extensive analysis of data for globular cluster RR Lyrae stars by Sandage (1982) has revealed a similar trend of $M_{v_{0}}$ with metallicity. (It should be noted, however, that statistical parallax analyses [see Clube and Dawe 1980] give a stronger trend with the opposite sign.) The RR Lyrae stars in the Magellanic Clouds (Graham 1973, 1975) have a mean visual absolute magnitude of +0.6 also. Thus our adopted values of $M_{v_{0}}$ for RR Lyrae stars as a function of metallicity are in agreement with the best available observational data and the predictions of pulsational theory, but must be regarded as uncertain by \pm 0.3 mag in their zero point.

\section{a) The Dependence of Giant Branch Location on}

$$
[\mathrm{Fe} / \mathrm{H}]_{\mathrm{Z}}
$$

Figure 2 shows $\log T_{e}(\mathrm{~GB})$ (the effective temperature of the mean giant branch of each cluster at $M_{\text {bol }}=-3.0$ ) versus $[\mathrm{Fe} / \mathrm{H}]_{Z}$ for the sample of 33 clusters. The circled points are the five globular clusters that lie farthest from the mean relationship between $(J-K)_{0}(\mathrm{~GB})$ and $[\mathrm{Fe} / \mathrm{H}]_{\mathrm{Z}}$ shown in Figure 3 . It is apparent from Figure 2 that $\log T_{e}(\mathrm{~GB})$ and $[\mathrm{Fe} / \mathrm{H}]_{\mathrm{Z}}$ are well correlated. The

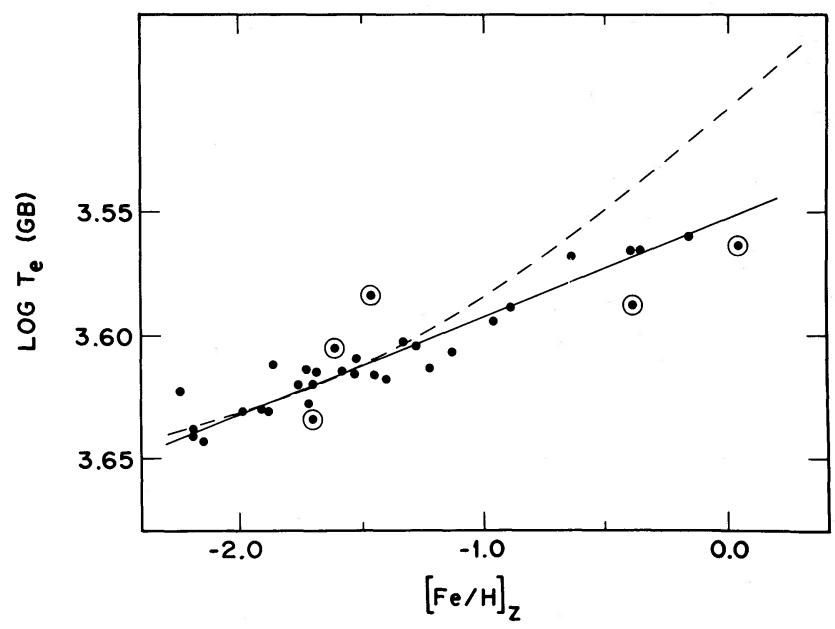

FIG. 2. $-\log T_{e}(\mathrm{~GB})$ is the effective temperature of a cluster GB read at $M_{\mathrm{bol}}=-3.0 .[\mathrm{Fe} / \mathrm{H}]_{\mathrm{Z}}$ is from Zinn's (1980) metallicity scale. The solid line is the least squares fit to the data found by averaging the two solutions found by taking first one and then the other quantity as the dependent variable. The dashed line is the correlation between the two quantities predicted by the Sweigart and Gross (1978) models for a mass of $0.7 M_{\odot}$ and a helium abundance $Y=0.3$. This line has been shifted by +0.03 in $\log T_{e}$ to agree with the data at the metal poor end. The circled points are clusters which lie off the sequence in Fig. 3. 


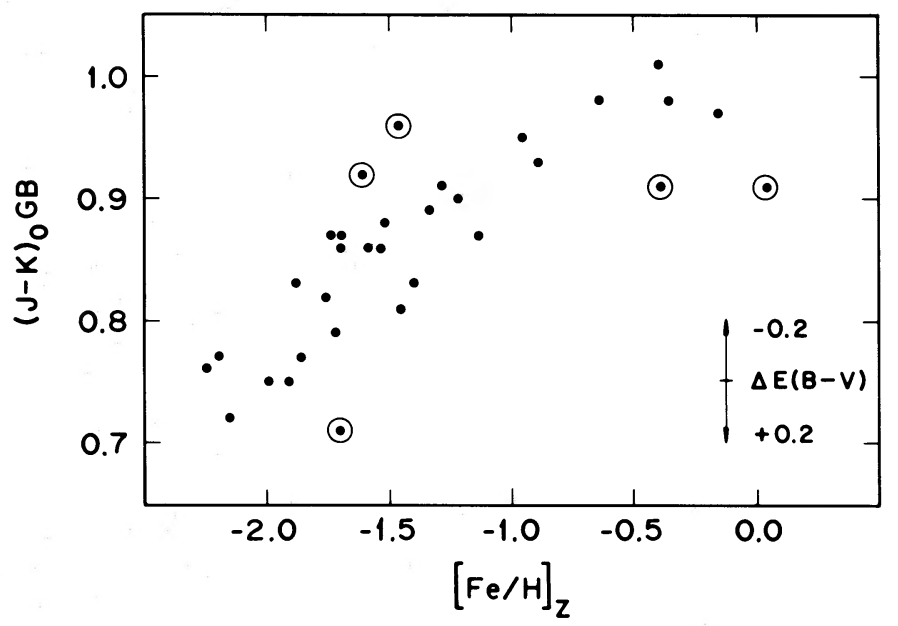

FIG. 3. $-(J-K)_{0} \mathrm{~GB}$ is the color of a cluster GB read at $M_{K_{0}}=-5.5$. The circled points are the clusters which lie farthest from a ridge line through the data, namely NGC 288, 4372, 6121 (M4), 6553, and 6637 (M69). The effect of a change in $E(B-V)$ of \pm 0.20 mag is shown in the lower right.

least squares solution obtained by solving for each quantity in turn as the independent variable and then averaging the results is

$$
[\mathrm{Fe} / \mathrm{H}]_{\mathrm{Z}}=89.92-25.32 \log T_{e}(\mathrm{~GB}) .
$$

The correlation coefficient $r$ is 0.92 and the dispersion in $\log T_{e}(\mathrm{~GB})$ is 0.009 -comparable to that expected from errors in the determination of $\log T_{e}(\mathrm{~GB})$ itself due to distance modulus and reddening uncertainties, the difficulty of placing a mean giant line through the data points, and the uncertainty in the $[\mathrm{Fe} / \mathrm{H}]_{\mathrm{Z}}$ scale. Thus a determination of $\log T_{e}(\mathrm{~GB})$ for a cluster should yield a metallicity, on Zinn's scale, of reasonable accuracy.

Figure 3 displays the dependence of $(J-K)_{0}(\mathrm{~GB})$ (the color of the mean GB of each cluster at $M_{K_{0}}=-5.5$ ) on $[\mathrm{Fe} / \mathrm{H}]_{\mathrm{Z}}$. Since $J-K$ is closely correlated with $V-K$ for metal-poor cluster stars (e.g., GC1, GC4) and $\log T_{e}$ is derived primarily from $(V-K)$, we would expect Figure 3 to show the dependence that it does. Unlike $V-K, J-K$ loses sensitivity to temperature below $4000 \mathrm{~K}$. One thus expects the relationship between $(J-K)_{0}(\mathrm{~GB})$ and $[\mathrm{Fe} / \mathrm{H}]_{\mathrm{Z}}$ to flatten out for high metallicities, and there is some evidence for this in Figure 3. However, for metallicities less than $-1.0 \mathrm{dex}$, the use of $(J-K)_{0}(\mathrm{~GB})$ as a metallicity indicator has the advantages over $\log T_{e}(\mathrm{~GB})$, which is based on $(V-K)_{0}(\mathrm{~GB})$, of utilizing observations made nearly simultaneously with the same detector system, being independent of potential systematic errors in photographic visual photometry and being less sensitive to reddening uncertainties.

The five circled points which lie farthest from the $(J-K)_{0}(\mathrm{~GB}),[\mathrm{Fe} / \mathrm{H}]_{\mathrm{Z}}$ mean sequence in Figure 3 are NGC 288, 4372, 6121, 6553, and 6637. The points for these clusters are circled in Figure 2 where they are also among the furthest from the mean sequence. Possible causes of these discrepancies will be discussed later.

Figure $4 a$ shows the dependence of $M_{K_{0}}(\mathrm{~GB})$ [the absolute, extinction-corrected, $K$ magnitude of the mean giant branch for each cluster at $\left.(V-K)_{0}=3.0\right]$ on $[\mathrm{Fe} / \mathrm{H}]_{\mathrm{Z}}$ together with the average of the two least squares solution. This line is given by

$$
M_{K_{0}}(\mathrm{~GB})=-3.15+1.09[\mathrm{Fe} / \mathrm{H}]_{\mathrm{z}} .
$$

The correlation coefficient is $r=0.86$, while the dispersion in $M_{K_{0}}(\mathrm{~GB})=0.36$. This dispersion is greater than expected from uncertainties in at least the relative abundance rankings and relative distance moduli. Since the giant branches of most of the clusters tend to slope steeply upward in infrared $C-M$ diagrams, the residuals from the mean relations between $M_{K_{0}}(\mathrm{~GB})$ and other quantities, are expected to be quite sensitive to errors, misclassifications, and other effects. The large dispersion is due primarily to two of the five clusters selected as being deviant in Figure 3 (M4 and M69), which are circled here, as before, plus NGC 2808 and 6397. The latter is also among the more deviant in Figure 2.

The dependence of $M_{K_{0}}(\mathrm{~GB})$ on $\log T_{e}(\mathrm{~GB})$ is seen to be exceptionally tight (Fig. 5). An $M_{\text {bol }}$ scale is indicated on the right side of the figure. An average of the least squares solutions yields

$$
M_{K_{0}}(\mathrm{~GB})=97.12-28.22 \log T_{e}(\mathrm{~GB})
$$

with a correlation coefficient $r=0.97$. The line drawn in Figure 5 is based on theory and is discussed below. The only two clusters identified as deviant in the previous figures which persist in such behavior here are NGC 2808 and 6121 (M4). Errors in the distance moduli and visual magnitudes will move the clusters parallel to the 


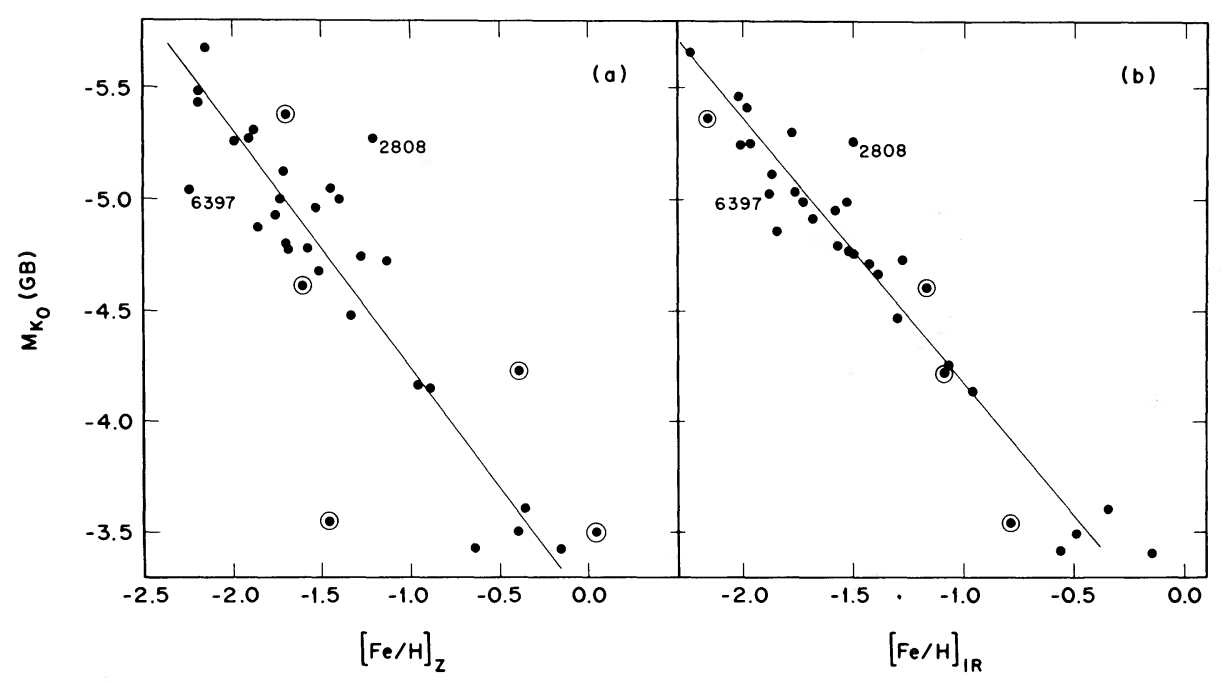

FIG. 4. - (a) $M_{K_{0}}(\mathrm{~GB})$ is the absolute $K$ magnitude of the cluster GBs read at $(V-K)_{0}=-3.0$. The circled points are from Fig. 3. NGC 2808 and 6397 are also identified. The solid line is the least squares fit determined as in Fig. 2. ( $b$ ) The same as Fig. $4 a$ except the infrared determined metallicity scale is used in place of Zinn's (1980) scale.

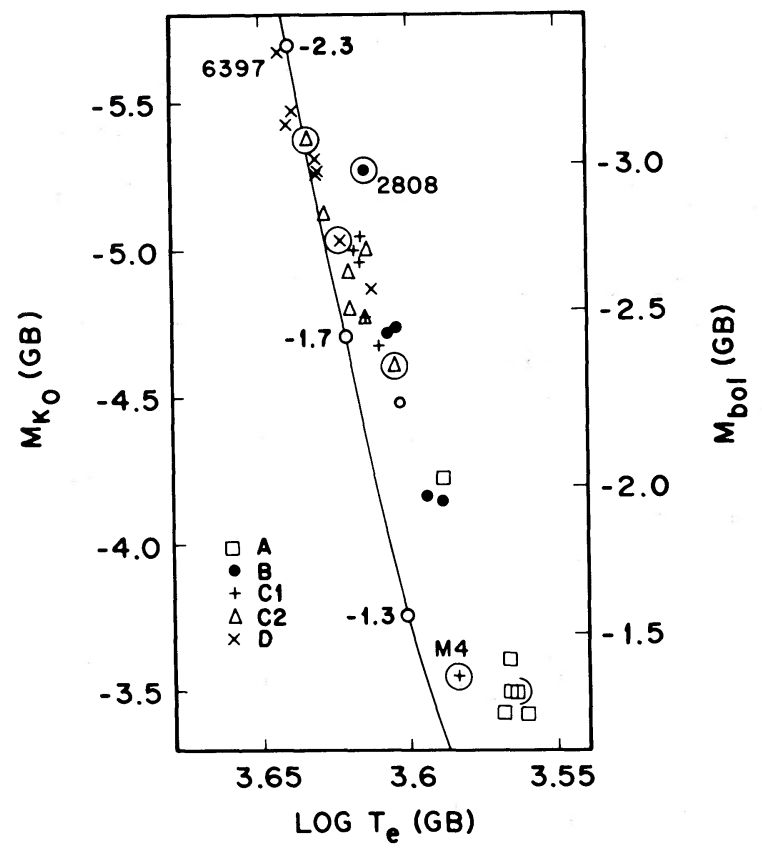

FIG. 5.-This shows the correlation between the infrareddetermined parameters of Figs. 2 and $4 a$. Only two of the clusters selected out in the previous figures (NGC 2808 and 6121) are significantly deviant from the rest. The solid line is the theoretical relation between $\log T_{e}(\mathrm{~GB})$ and $M_{\mathrm{bol}}(\mathrm{GB})$ derived as discussed in the text. The locations of these models with $[\mathrm{Fe} / \mathrm{H}]_{\text {solar }}=-2.3$, -1.7 , and -1.3 are shown. mean line in Figure 5. Errors in $E(B-V)$, on the other hand, will act to move the points in Figure 5 nearly vertically - an error of 0.07 in $E(B-V)$, shifts a point by -0.006 to -0.009 in $\log T_{e}(\mathrm{~GB})$, but by 0.3 to 0.4 in $M_{\text {bol }}(\mathrm{GB})$. We thus have a strong case for two highly suspect values of $E(B-V)$, namely those of M4 and NGC 2808. We would argue, on the basis of Figure 5, that for M4 $E(B-V)$ should be changed from 0.36 to about 0.45 and for NGC 2808 from 0.22 to about 0.15 mag. Other arguments supporting a higher value of $E(B-V)$ for M4 are given in $\S \mathrm{IV} a$ and VI $e$ of GC8.

The tight relationships of Figures $2-5$ that exist between two parameters which characterize the horizontal and vertical positioning of the giant branches demonstrate that globular cluster giant branches are strictly homologous along a metallicity sequence.

\section{b) Comparison with Theory}

Calculations of stellar interior models (e.g., Sweigart and Gross 1978; Rood 1972) predict that if other physical parameters are kept constant, the ordering of globular cluster giant branches in $T_{e}$ at constant luminosity or in luminosity at constant $T_{e}$ should depend only on the abundance of metals heavier than the CN0 group. If we accept Zinn's (1980) $[\mathrm{Fe} / \mathrm{H}]$ ranking for the clusters, then Figures 2-4 are an important confirmation of the prediction. Alternatively, if the theoretical result is accepted $a$ priori, then these figures demonstrate that a metallicity ranking scheme based on infrared observations of individual giant stars is consistent to within 0.2 dex in $[\mathrm{Fe} / \mathrm{H}]$ with a scheme based on integrated light observations of line and molecular blanketing in the blue region of the spectrum. Although a metallicity scale derived from $\log T_{e}(\mathrm{~GB})$ is to some extent dependent on 
a knowledge of the cluster distances, it in fact requires only that the absolute magnitude of the RR Lyrae stars be independent of, or a monotonic function of, $[\mathrm{Fe} / \mathrm{H}]$.

In spite of the agreement in ranking between theory and observation, in GC5 we presented evidence based on a limited number of clusters that there is a scaling problem with theoretical GB temperatures in that to bring theoretical GBs into agreement with observed ones, it was necessary to shift the theoretical branches in temperature by an amount which was a function of $[\mathrm{Fe} / \mathrm{H}]$. With the large sample of clusters now observed, the discrepancy can be quantified. In Figures 2 and 5 we have indicated the theoretical relationship between $\log T_{e}$ at $M_{\text {bol }}=-3.0$, metallicity, and $M_{\text {bol }}$ at a $T_{e}$ corresponding to $(V-K)_{0}=3.0$ for giant stars with $M=0.7$ $M_{\odot}$ and $Y=0.3$ from the tracks of Sweigart and Gross (1978). A uniform shift of +0.03 in $\log T_{e}$ has been made to force agreement with the observations at the metal-poor end of the sequence. (This is the same shift used for 47 Tuc in GC5.) In spite of this, as shown in Figure 2, the models get progressively cooler than the observations at higher metallicities. Possible problems with the $(V-K)_{0}$ temperature scale for the coolest stars, discussed in GC8, cannot eliminate the discrepancy between theory and observation; use of the $(J-K)_{0}$ scale exacerbates the disagreement. If the absolute visual magnitudes of RR Lyrae stars increase between the metal-poor and metal-rich clusters by twice the $+0.2 \mathrm{mag}$ assumed here, the effect on the empirical $\log T_{e}(\mathrm{~GB})$ 's at the metal-rich end would be a shift of only -0.009 which is not enough to remove the discrepancy. A change in the $Y$ value for the models tends to shift the theoretical line up and down in toto.

In GC5 we concluded that the most probable source of the overall 0.03 shift in $\log T_{e}$ required to bring theory and observation into agreement was a slightly wrong initial choice of $\alpha$, the mixing length to scale height ratio in the models. Equation 2.10 of Renzini (1977) for giant branch stars gives $\Delta \log T_{e}=+0.2 \Delta \log \alpha$. Hence a systematic change in $\alpha$ by a factor of no more than 1.5 over the observed range of $\mathrm{Fe} / \mathrm{H}$ would be sufficient to remove the discrepancy in Figure 2. Recent model calculations appropriate to globular clusters by VandenBerg (1983) show that for stars near the main-sequence turnoff and on the subgiant branch, an increase in $\alpha$ has a significantly greater effect on high metal abundance stars than on low. If this result is found to hold true for GB stars as well, then a metallicity-dependent $\alpha$ would not be required. It would just be necessary to start out with a value larger than that used by Sweigart and Gross (1978), viz., $\alpha=1$.

\section{c) The Luminosity of the Tip of the Giant Branch}

A relationship between the bolometric luminosity of the brightest red giant in a globular cluster and that cluster's metallicity, as well as the close agreement be- tween this relationship and the theoretical dependence of the luminosity of a star at core helium flash on metallicity, was pointed out by Frogel, Persson, and Cohen (1981b).

Figure $6 a$ is a plot of the absolute bolometric magnitude of the brightest star in each of the clusters observed against $[\mathrm{Fe} / \mathrm{H}]_{\mathrm{Z}}$. Known LPVs in four clusters have been excluded from this plot since it is quite likely that they are AGB stars rather than first ascent giants (see, for example, Wood and Cahn 1977). (The excluded stars are V1-4 in NGC 104, V3 in NGC 5927, V4 and V5 in NGC 6553, and V4 in NGC 6637.) The properties of the variables are discussed in Frogel (1983). First we note that when the LPVs are excluded, none of the red giant stars which have been measured is brighter than $M_{\text {bol }}=$ -3.82. Second, there is clearly a correlation between $M_{\text {bol }}(1 \mathrm{st})$ and $[\mathrm{Fe} / \mathrm{H}]_{\mathrm{Z}}$. The least squares fit is shown as a solid line in Figure $6 a$. It was noted in GC8 that for 11 of the 33 clusters observed it is possible, because of lack of infrared scans of the cluster core and incompleteness of optical surveys there, that the 1st brightest star may not in fact have been observed. These clusters are plotted with open circles in Figure 6. The open circles do not show any propensity for the fainter part of the distribution, and importantly, if the two points with the largest values of $M_{\text {bol }}(1 \mathrm{st})$ at large $[\mathrm{Fe} / \mathrm{H}]_{\mathrm{Z}}$. were to be replaced with brighter stars, the $M_{\text {bol }}$ $(1 \mathrm{st}) /[\mathrm{Fe} / \mathrm{H}]_{Z}$ slope would only steepen.

The luminosity of the brightest giant found in a cluster will depend not only on observational selection but also on the total number of stars in the cluster. The upper part of cluster giant branches are sparsely populated, so that a significant reduction in cluster richness will bias the luminosity of the brightest star downward. As a measure of richness we have taken the integrated $V$ magnitudes tabulated by Harris and Racine (1979) and adjusted them for the values of $E(B-V)$ and $(m-M)_{0}$ in use here. Integrated $M_{V_{0}}$ 's for our sample of clusters range from -5.4 for NGC 6838 (M71) to more than -9.2 for NGC 104 and 2808. We single out the six faintest clusters (NGC 6352, 6838, 6362, 288, 2298, and 6397) and also NGC 6553 because the severe crowding of the field and lack of an infrared survey has certainly led to an incomplete survey of stars near the tip of the giant branch (GC8). These seven clusters are noted with plus signs in Figure 6. Five of the seven lie near the faint edge of the distribution, as expected.

Fitting a least squares line through the remaining 26 clusters yields the relationship

$$
M_{\mathrm{bol}}(1 \mathrm{st})=-3.82-0.26[\mathrm{Fe} / \mathrm{H}]_{\mathrm{Z}}, r=0.79, \sigma=0.10
$$

shown as a dashed line in Figure $6 a$. For these 26 clusters there is no significant correlation between the residuals in $M_{\text {bol }}$ (1st) and $M_{V_{0}}$ (integrated). 


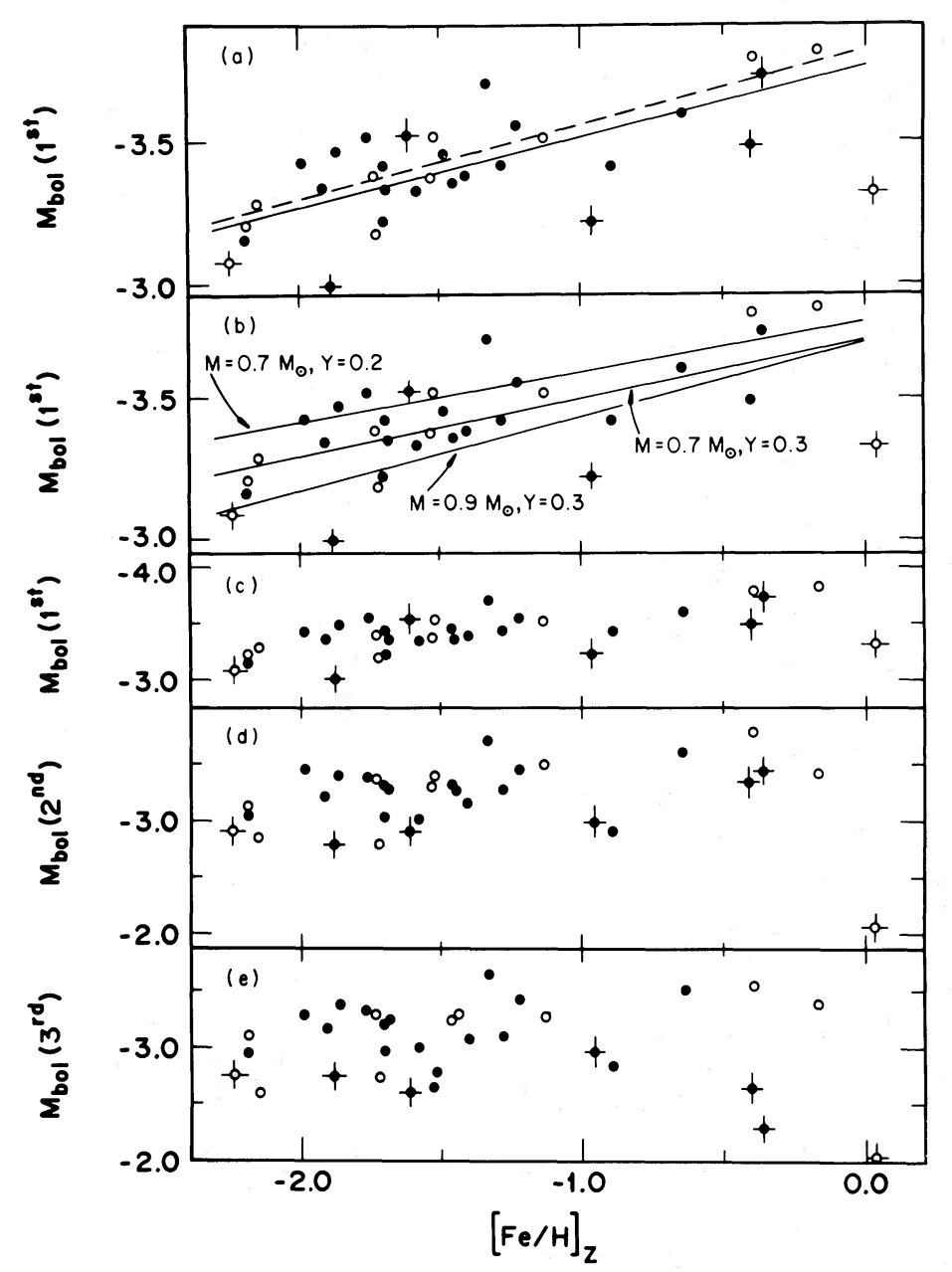

Fig. 6. - The bolometric magnitude of the brightest star in each cluster as a function of metallicity. Points with pluses through them are from the six least populous clusters and NGC 6553 . The solid line is the least squares fit to the data determined by taking only $M_{\text {bol }}$ (1st) as the dependent variable. NGC 6553 has been excluded for reasons discussed in the text. The dashed line in the fit determined by excluding NGC 6553 and the six least populous clusters. (b) Same data as in Fig. $6 a$, but now the dependence of the tip of the GB (presumably the location of the core He flash) on metallicity for three model sequences from Sweigart and Gross (1978) is shown. Note that these theoretical lines have not been shifted along either axis before being plotted. (c) Same data as in Fig. $6 a$ but on a reduced scale. ( $d$ and $e$ ) Same as Fig. $6 c$, but for the second and third brightest stars (bolometrically) in each cluster.

Since we are confident of having observed the 2 nd and 3 rd brightest stars in the two-thirds of our sample that has been surveyed into the clusters' centers (see Table 29 of GC8), their dependence on cluster metallicity can be investigated as well. Their bolometric magnitudes are plotted in Figures $6 d$ and $6 e$. There is no statistically significant dependence of the magnitudes of these stars on metallicity; the formal dispersions are 0.23 and $0.32 \mathrm{mag}$, respectively, nearly 2 and 3 times greater than that found for the first brightest star.

It is a rather remarkable result that the small dispersion (0.1 mag) found for $M_{\text {bol }}$ (1st) in equation (4) is only half of what we would have conservatively predicted the scatter in absolute magnitudes to be due to uncertainties in the distance scale alone. Independent of the origin of this small dispersion, which we discuss below, we have important empirical confirmation of the widely held assumption that the intrinsic scatter in the absolute magnitudes of horizontal branches is indeed small-not more than $0.1 \mathrm{mag}$ - for clusters of similar metallicity.

The empirical relation between cluster metallicity and $M_{\text {bol }}$ (1st) is exceptionally close in both level and slope to the theoretically predicted relation between metallicity and the luminosity at the core helium flash in first ascent giants. Three theoretical sequences from the Sweigart and Gross (1978) models are drawn in Figure $6 b$. We emphasize that uncertainties in convective the- 
TABLE 2

Monte Carlo Calculations for First Brightest Star

\begin{tabular}{cccc}
\hline \hline \multicolumn{1}{c}{$\begin{array}{c}P(0.1) \\
N_{\mathrm{RG}}\end{array}$} & $\begin{array}{c}P(0.1) \\
\text { (no AGB) }\end{array}$ & $N_{\mathrm{AGB}}$ & (with AGB) \\
\hline $9 \ldots \ldots$. & 0.07 & 3 & 0.18 \\
$20 \ldots \ldots$ & 0.16 & 7 & 0.42 \\
$40 \ldots \ldots$ & 0.26 & 14 & 0.68 \\
$60 \ldots \ldots$ & 0.42 & 20 & 0.79 \\
$80 \ldots \ldots$ & 0.50 & 27 & 0.86 \\
\hline
\end{tabular}

ory will affect only the temperatures of the models, not the luminosities. Similar agreement holds for the models of Rood (1972).

In Frogel, Persson, and Cohen (1981b) we suggested that the dispersion in $M_{\text {bol }}$ (1st) was smaller than expected and that this could be due to some neglected physical effect which slows down the rate of stellar evolution near the tip of the giant branch. We now reconsider this in more detail and suggest instead that the small dispersion is to be expected statistically. The contribution of AGB stars close to the tip of the giant branch is uncertain. In those cluster $C-M$ diagrams where the AGB can be clearly seen, it merges with the RGB well below the tip, and we consequently have no way of being certain that mass loss is effective in removing these (lower mass) stars before they reach the tip, or whether they are contaminating the region of the tip at a significant level. The luminosity distribution of AGB stars is flat (in $M_{\text {bol }}$ ) (Iben and Truran 1978), while the RGB distribution falls steadily as the stellar evolutionary rates accelerate approaching the tip; thus, AGB stars in principle could outnumber the RGB stars close to the tip.

In order to compare theoretical distributions of stars near the tip with those observed, we have carried out a series of Monte Carlo calculations. Luminosity distributions were predicted for a range in cluster richness, parameterized by the number of red giants within a 2 bolometric magnitude interval below the tip, (denoted $N_{\mathrm{RG}}$ ) using the evolutionary sequences in Sweigart and Gross (1978), both with and without a one-third (by number) contribution from AGB stars. The probabilities of finding the first, second, etc. brightest star were found as a function of magnitude below the tip. Representative results for the 1st brightest star being within $0.1 \mathrm{mag}$ of the tip $P(0.1)$ both with and without AGB stars included are given in Table 2.

Two important features of Table 2 are apparent. First, the AGB contribution is crucial, with the probability that the 1st brightest star lies within $0.10 \mathrm{mag}$ of the tip more than doubling for the sparser clusters when the AGB stars are included. The different shapes of the AGB and RGB luminosity functions account for this effect. Second, the probability that the 1st brightest star lies within $0.1 \mathrm{mag}$ of the tip rises to more than $50 \%$ for clusters with as few as 40 stars in the 2 mag interval below the tip if the AGB contribution is included. This is certainly qualitatively consistent with the appearance of Figure $6 a$ if the tip is actually the upper envelope of points. We will show below that it is also quantitatively consistent. Thus in this case, our data could be interpreted to favor a helium abundance near 0.2 for globular clusters (or systematically fainter RR Lyrae magnitudes and smaller distances than those adapted here).

To compare the Monte Carlo probabilities of Table 2 with the clusters of Figure 6, we must establish values for the parameter $N_{*}$, the number of stars within 2 bolometric mag of the red giant tip for each cluster. This was done by examining the sample for each cluster from which the stars observed in the infrared were chosen (described in GC8). The original total samples in $V$, $B-V C-M$ diagrams were converted to limits in $M_{\mathrm{bol}}$ and summed to derive $N_{*}$ in the 2 bolometric magnitude interval below the tip. The tip was assumed to occur at that luminosity predicted by the Sweigart and Gross track for a $0.7 M_{\odot}$ star with $Y=0.3$ and the appropriate metallicity of the cluster. Table 3 lists the deduced distribution of $N_{*}$. Although the values of $N_{*}$ are crude, a value of 20 is typical, while 40 is an upper limit even for a very rich cluster. Table 2 thus implies that if the AGB does extend all the way to the red giant tip, for the average cluster, the probability is $30 \%$ that the brightest star will be within $0.1 \mathrm{mag}$ of the tip. For the richest clusters, the probability of this occurring is more than $80 \%$.

The Monte Carlo calculations thus demonstrate that the distribution in magnitude of the 1st brightest star observed in our sample of globular clusters is consistent with that expected from statistics if the AGB extends all the way up the giant branch to the tip. The Monte Carlo calculations also reproduce satisfactorily the observed behavior of $M_{\text {bol }}(2 \mathrm{nd})$ and $M_{\mathrm{bol}}(3 \mathrm{rd})$ displayed in the lower panels of Figure 6 . We emphasize though the uncertainty in these Monte Carlo calculations arising from the poorly known mass loss rates for AGB stars and thus the uncertain luminosity at which the AGB terminates. The presence of luminous long-period variables in metal-rich clusters suggests (e.g., GC5 and Frogel 1983) that in at least these clusters, AGB evolution can proceed beyond the maximum luminosity of

TABLE 3

Cluster RichNesS Distribution

\begin{tabular}{|c|c|}
\hline$N_{*}(2$ mag below tip $)$ & No. of Clusters \\
\hline $5-10 .$. & 3 \\
\hline $11-15$. & 6 \\
\hline $16-20 \ldots$ & 8 \\
\hline $21-25 \ldots$ & 5 \\
\hline $26-30 \ldots$ & 2 \\
\hline $31-35 \ldots$ & 6 \\
\hline
\end{tabular}


the first ascent red giants. If the most luminous stars in the majority of the sample clusters are actually AGB stars, it would seem somewhat fortuitous that the dependence of their luminosity on metallicity should so closely mimic that expected for first ascent red giants. (Recall that only four clusters, all metal-rich, have long-period variables that have been removed from the sample.)

Irrespective of its origin, the rather tight correlation between $M_{\text {bol }}(1 \mathrm{st})$ and $[\mathrm{Fe} / \mathrm{H}]$ can be useful as an accurate distance indicator to more distant Population II clusters and stellar systems. A rough estimate of $[\mathrm{Fe} / \mathrm{H}]$ should result in $(m-M)_{0}$ values with an accuracy of $0.1-0.2 \mathrm{mag}$. For a system with a range in stellar ages, the break in slope of the luminosity function caused by the GB ending and only the AGB (where the number of stars per unit $M_{\text {bol }}$ is constant, unlike the GB) stars remaining, may prove useful. Much of this work could be done with the Space Telescope by carefully calibrated observations in the $0.7-1.0 \mu \mathrm{m}$ range. $^{2}$

These conclusions concerning $M_{\text {bol }}$ (1st) depend critically on the distance scale employed in this series of papers, reviewed in the beginning of this section. If $M_{v_{0}}$ for the horizontal branch in metal-rich clusters is closer to +1.0 rather than the 0.8 assumed here, or if $Y$ is somewhat smaller than 0.3 , inspection of Figures $6 a$ and $6 b$ shows that the agreement between the theoretical and empirical relations will in no case be degraded, but can only be improved. Furthermore, agreement with the Monte Carlo calculations without invoking the presence of AGB stars near the tip can also be achieved. One can invert the whole argument if desired, and assume that the theoretical luminosity for the helium flash is in fact correct, and then use Figure 6 to determine how $M_{v_{0}}$ for RR Lyrae stars must vary with metallicity to produce consistency. Such a point of view strongly suggests that our assumed metallicity dependence for $M_{v_{0}}$ of the RR Lyrae stars is correct to within \pm 0.1 mag over the whole metallicity range. Furthermore, any normalization where the metal-poor RR Lyrae stars are more luminous than $M_{v_{0}}=+0.6$ runs into serious difficulties, while if $M_{v_{0}}$ is taken as fainter by +0.2 mag (as suggested by Sandage 1982), then few clusters actually have any star as bright as the helium flash.

\section{CO ABSORPTION AND CLUSTER HORIZONTAL BRANCHES}

\section{a) Correlation of $\mathrm{CO}(G B)$ with $[\mathrm{Fe} / \mathrm{H}]_{\mathrm{Z}}$}

There is a good correlation between $\mathrm{CO}(\mathrm{GB})$ [the median $\mathrm{CO}$ in the neighborhood of $\left.(V-K)_{0}=3.0\right)$

\footnotetext{
${ }^{2}$ The error introduced by missing the 1 st brightest star but finding the 2 nd or 3 rd brightest is not too large. In the sample of clusters from which the six sparest ones have been eliminated, we find that $\Delta M_{\text {bol }}$ (2nd-1st) is greater than $0.2 \mathrm{mag}$ in only six out of 26 cases, and $\Delta M_{\text {bol }}$ (3rd-1st) is greater than 0.3 mag in only seven out of 26 cases.
}

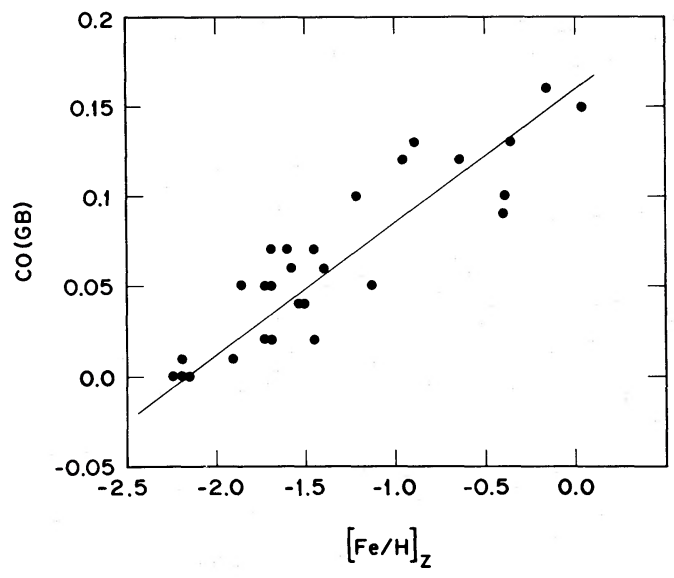

Fig. 7. $-\mathrm{CO}(\mathrm{GB})$ is the median value of the $\mathrm{CO}$ index for the stars in a cluster with $(V-K)_{0}$ near 3.0. The solid line is the least squares fit determined as for Fig. 2.

given in column (13) of Table 1 and $[\mathrm{Fe} / \mathrm{H}]_{Z}$ as may be seen in Figure 7. The average of the two least squares fits gives

$$
\mathrm{CO}(\mathrm{GB})=0.16+0.074[\mathrm{Fe} / \mathrm{H}]_{\mathrm{Z}}
$$

with $r=0.90$ and a dispersion in $\mathrm{CO}(\mathrm{GB})$ of 0.02 . The uncertainties in the $\mathrm{CO}$ observations themselves are no more than 0.015 mag while the spread in $\mathrm{CO}$ near $(V-K)_{0}=3.0$ in a typical metal-poor cluster is at least $0.03 \mathrm{mag}$, giving rise to most of the apparent dispersion in Figure 7. Nevertheless, there are several pairs of clusters in Table 1 , each member of the pair having similar metallicities, values of $(V-K)_{0}(\mathrm{~GB})$ and $(J-$ $K)_{0}(\mathrm{~GB})$, but whose stars have strongly systematic differences in their $\mathrm{CO}$ strengths. Examples are M3 and M13 (GC1 and Pilachowski 1978) and M22 and any other cluster in group D of Table 1 (see the detailed discussion of M22 in $\S \mathrm{IVg}$ of GC8). The general effect of a spread in $\mathrm{CO}$ is discussed below.

As expected, $\mathrm{CO}(\mathrm{GB})$ correlates well with $\log T_{e}(\mathrm{~GB})$; the latter gives a completely independent metallicity ranking for the clusters. The relationship between these quantities is given by

$$
\mathrm{CO}(\mathrm{GB})=7.06-1.94 \log T_{e}(\mathrm{~GB}),
$$

with $r=0.88$ and a dispersion in $\mathrm{CO}(\mathrm{GB})$ of 0.023 .

Note that a few clusters have values of $\mathrm{CO}(\mathrm{GB})$ comparable to or greater than the 0.13 found for solar neiGBborhood field giants of $(V-K)=3.0$ (Frogel et al. 1978). It remains to be seen if this reflects a truly enhanced molecular abundance or is the by-product of molecular blanketing effects. At $(V-K)_{0}=3.0$, which corresponds to $T_{\text {eff }}=4300 \mathrm{~K}$, we expect the complete absence of $2.2 \mu \mathrm{m} \mathrm{CO}$ absorption to result in a $\mathrm{CO}(\mathrm{GB})$ $=-0.04$ Since the minimum observed value is 0.00 , 
even the most metal-poor cluster giants probably have some $\mathrm{CO}$ absorption.

Thus for globular cluster giants of constant $(V-K)_{0}$ (i.e., temperature), Figure 7 shows that the $\mathrm{CO}$ absorption strength is a monotonically increasing function of metallicity with some real scatter superposed. ${ }^{3}$ For field stars $\mathrm{CO}$ is a generally increasing function of luminosity (Baldwin, Frogel, and Persson 1973). It is thus interesting that the increase in $\mathrm{CO}$ with metallicity overcomes the expected decrease due to decreasing stellar luminosity at constant color (which amounts to about 2 mag in $M_{K_{0}}$ [Fig. 4] or $M_{\text {bol }}$ ).

\section{b) $\mathrm{CO}(G B)$ Residuals and the Correlation with Horizontal-Branch Type}

The first observation of globular cluster stars in the infrared (GC1 and Pilachowski 1978) led to the suggestion that cluster to cluster $\mathrm{CO}$ variations at constant metallicity were related to horizontal-branch morphology, and hence that the CNO element group had been identified as the "second parameter." Subsequent observations of $\omega$ Cen and NGC 7006 (GC3 and GC7) cast doubt on this interpretation. The present cluster sample is large enough to investigate this question in a definitive manner. The full range of horizontal-branch (HB) types is represented and, as may be seen from Figure $8 a$ (Fig. $8 b$ will be discussed in $\S \mathrm{VI}$ ), the intermediate metallicity clusters alone show almost the full spread. Now $\mathrm{CO}(\mathrm{GB})$ does show a correlation with $\mathrm{HB}$, but this is only to be expected from the correlation of each of these parameters with metallicity, as shown in Figures 7 and 8 . What must be examined for each cluster is the deviation of $\mathrm{CO}(\mathrm{GB})$ from the mean relationship of Figure 7 (denoted $\mathrm{CO}-\langle\mathrm{CO}\rangle$ ) as a

\footnotetext{
${ }^{3}$ The scatter remains when $\mathrm{CO}(\mathrm{GB})$ is plotted as a function of the revised metallicity scale defined in $\S \mathrm{VI}$.
}

function of the deviation of $\mathrm{HB}$ from the mean $\mathrm{HB}$ type at each $[\mathrm{Fe} / \mathrm{H}]_{Z}$ (denoted $\mathrm{HB}-\langle\mathrm{HB}\rangle$ ). This is displayed in Figure 9. It is obvious that even for clusters belonging to the same metallicity group there is no correlation between the $\mathrm{CO}(\mathrm{GB})$ and $\mathrm{HB}$ residuals. The early indication of a correlation was due mostly to M3 and M13 which are marked in Figure 9. Thus the CO abundance is unrelated to the second parameter problem.

\section{c) The Spread in $\mathrm{CO}(G B)$}

We now consider the spread in $\mathrm{CO}$ absorption at a given color within each cluster. The spread observed in 47 Tuc could be satisfactorily explained (GC5) as due to the effects of star to star variations in carbon and nitrogen abundances and in the resulting $\mathrm{CN}$ opacity. Enough stars have been observed in many of the clusters in the present sample that the prevalence of a range in

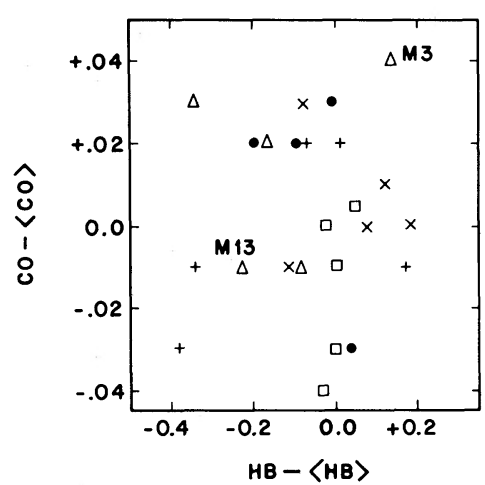

FIG. 9. - The $\mathrm{CO}$ residuals from the fit in Fig. $7 a$ are plotted against the H.B. Parameter residuals of Fig. 8. Clusters in the five different metallicity groups of Table 1 are distinguished by different symbols which are identified in Fig. 5.

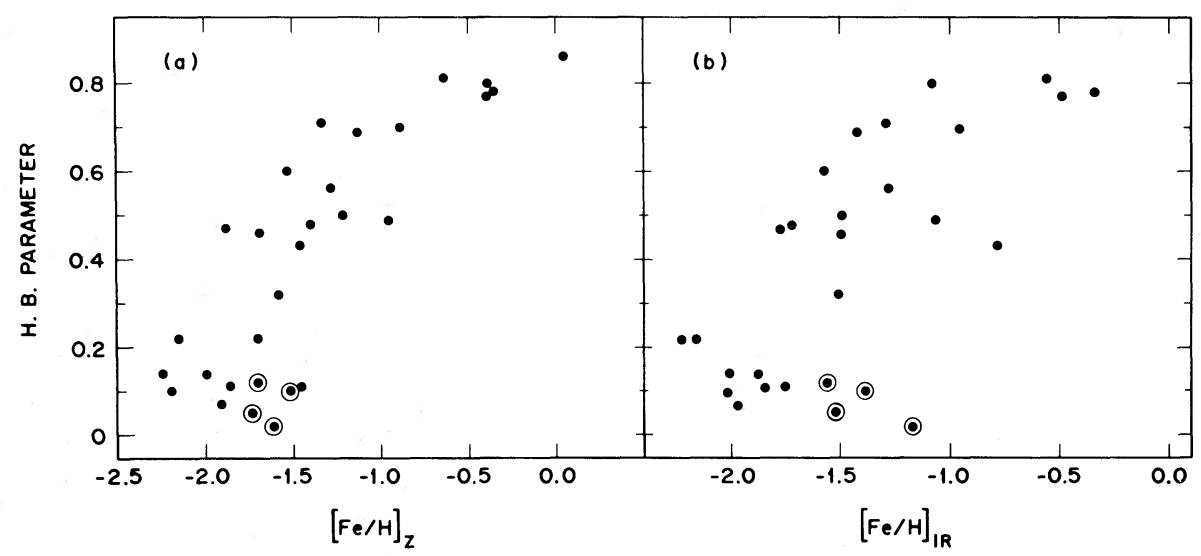

Fig. 8. - The H.B. Parameter is the fraction of red horizontal branch stars. The circled points are M10, M13, NGC 288, and 6752-four clusters with exceptionally blue HBs for their metallicity as discussed in the text. 


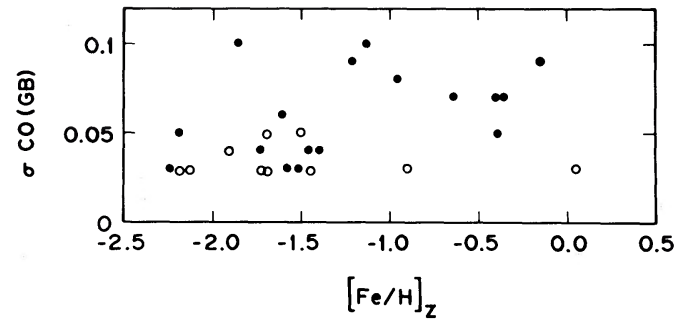

FIG. 10. $-\sigma(\mathrm{CO})$ is the spread in $\mathrm{CO}$ for each cluster as determined from stars with $(V-K)_{0}$ between 3.0 and 3.5. The open circles denote those clusters with less than 10 stars with measured $\mathrm{CO}$ indices.

$\mathrm{CO}$ strengths can be examined. The parameter $\sigma(\mathrm{CO})$ [the spread of the observed stellar $\mathrm{CO}$ values in the region of the GB with $\left.(V-K)_{0}=3.0-3.5\right]$ should give an indication of the spread from cluster to cluster, and Figure 10 shows its behavior as a function of $[\mathrm{Fe} / \mathrm{H}]_{\mathrm{Z}}$. The open symbols in this figure denote clusters where the total number of observed stars is less than or equal to 10 , so that $\sigma(\mathrm{CO})$ may be underestimated.

The clusters in Figure 10 seem to fall into two groups. The metal-rich clusters with $[\mathrm{Fe} / \mathrm{H}]_{\mathrm{Z}}>-1.0$ tend to have $\mathrm{CO}$ spreads that are greater than the measurement uncertainties and are comparable to 47 Tuc with its spread of $0.07 \mathrm{mag}$. The metal-poor clusters with $[\mathrm{Fe} / \mathrm{H}]<-1.3$, except $\mathrm{M} 22$, have spreads which in general can be attributed to measuring uncertainties and fluctuations due to the small numbers of stars observed in some cases. The three clusters of the total sample with the largest values of $\sigma(\mathrm{CO})$ are NGC 2808, 362, and 6656 (M22), two of which are in metallicity group B and one in group D. [The value of $\sigma(\mathrm{CO})$ for $\omega$ Cen (GC3) where a significant range in heavy metal abundance among its members undoubtedly exists, would be 0.10-0.12.] In the Appendix to GC8, we demonstrated that strong $\mathrm{CN}$ variations exist in two of these three clusters. (There are no suitable observations of molecular band strengths for NGC 2808). Such CN variations, bimodality, and CO-CN anticorrelations are also known to exist in M71 and 47 Tuc. The remaining group $\mathrm{A}$ clusters all have $\sigma(\mathrm{CO})$ values about equal to those of 47 Tuc and $\mathrm{M} 71$, in both of which $\sigma(\mathrm{CO})=0.07 \mathrm{mag}$, except for two clusters where only a small sample of stars was observed. It may well be that all the metal-rich clusters will show the same phenomenon when molecular band strengths are available for enough stars. The larger values of $\sigma(\mathrm{CO})(0.09-0.10 \mathrm{mag})$ in the three metal-poor clusters cited above may be due to the decreased importance of saturation in the molecular bands, rather than to a larger variation than in the group $\mathrm{A}$ clusters in actual $\mathrm{CN}$ and $\mathrm{CO}$ abundances.

\section{COMPARISON WITH INTEGRATED LIGHT INFRARED PHOTOMETRY}

Integrated infrared colors and $\mathrm{CO}$ indices for globular clusters have been discussed in Frogel et al. (1978),
Aaronson et al. (1978, hereafter ACMM), and Frogel, Persson, and Cohen (1980). These studies have demonstrated that the integrated properties are strong functions of cluster metallicity. Although it is certain that a significant fraction of the integrated light at $2.2 \mu \mathrm{m}$ is coming from the cluster GB stars, we examine the correlation between colors and indices determined by observing individual stars with those found from the integrated light observations as an important consistency check.

Thirteen clusters are common to ACMM and the present work, 12 of which have $\mathrm{CO}$ measurements in both studies. We took the ACMM data and applied the reddening corrections used here (which in a few cases differed significantly from the values they used ${ }^{4}$ ). Figures $11 a$ and $11 b$ display the correlations between ( $V-$ $K)_{0}(\mathrm{~GB})$ and $\mathrm{CO}(\mathrm{GB})$ with the corresponding quantities from the integrated light photometry (denoted by ACMM). ACMM quote uncertainties of 0.025 and 0.15 mag for the $\mathrm{CO}$ and $V-K$ integrated light measurements, respectively; these can account for the dispersions entirely. As would be expected from dilution of the giant branch contribution to the total integrated light, the slopes in Figure 11 are somewhat greater than unity. Also shown as open circles connected by a solid line are the corresponding points representing the theoretical models of the integrated light of globular clusters used by ACMM. The $(V-K)_{0}(\mathrm{~GB})$ and $\mathrm{CO}(\mathrm{GB})$ values were extracted from the GB tracks used by ACMM to construct the integrated light models, and the integrated light values are those calculated for a Salpeter mass function.

This comparison demonstrates that integrated infrared colors and indices of globular clusters are quite sensitive to the same quantities as are the individual stellar data, namely, the effective temperature of the giant branch and the $\mathrm{CO}$ absorption strengths of the stars on the GB. The constituents of the integrated light of globular clusters, at least from $V$ to $K$, are well understood.

\section{A SUGGESTED REVISION OF THE METALLICITY SCALE}

Our cluster GB parameters can, as described in §§ III and IV, be used to rank globular clusters in metallicity based on the relative location of the giant branches in the $\mathrm{H}-\mathrm{R}$ diagram. Theoretical calculations reviewed in $\S$ III suggest that this ranking will be sensitive only to the abundance of the heavy elements. The giant branch parameters change smoothly as a function of $[\mathrm{Fe} / \mathrm{H}]_{\mathrm{Z}}$ over the entire metallicity range spanned by the sample of 33 globular clusters as shown in Figures 2-4. Even at the metal-rich end, there is no apparent abrupt change

\footnotetext{
${ }^{4}$ Application of the reddening corrections from GC8 rather than from ACMM increased the correlation coefficient and reduced the dispersion in the quantities compared.
} 

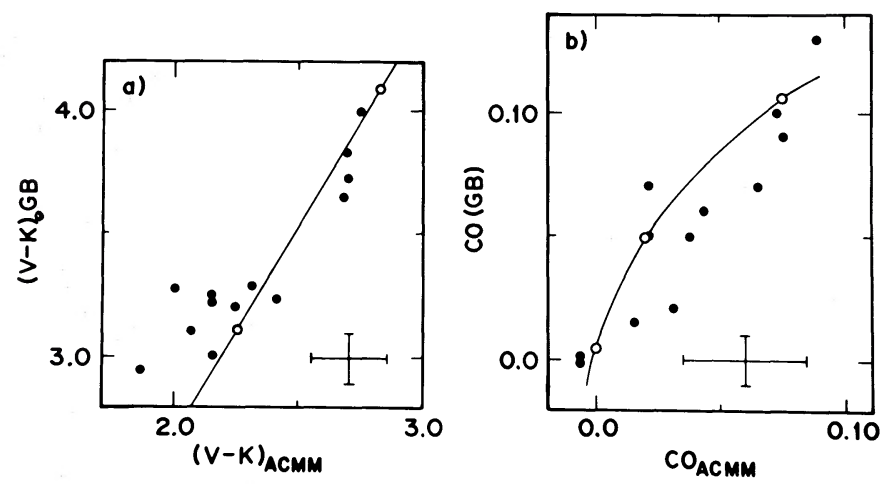

FIG. 11.- The correlation between $(V-K)_{0}(\mathrm{~GB})$ and $\mathrm{CO}(\mathrm{GB})$ determined from observations of individual stars in clusters (Table 1 here) and the integrated light data from ACMM, modified as discussed in the text. The solid lines connect the model points (denoted by open circles) as discussed in the text. The $\pm 1 \sigma$ error bars do not include reddening uncertainties.

in slope of the mean relationships. This is evidence against the analyses of Pilachowski, Sneden, and Green (1981) who suggest that many intermediate and high metallicity clusters, including NGC 3201, 47 Tuc, and NGC 6352 have essentially the same abundance, $[\mathrm{Fe} / \mathrm{H}]$ $=-1.1 \pm 0.2 \mathrm{dex}$. The reader is referred to $\S \mathrm{VI}$ of GC8, where a graphical comparison of the giant branches of a number of these clusters is given.

A ranking based on the giant branch parameters should, in some respects, be superior to that of Zinn (1980), as the former is based on photometry of individual stars rather than integrated light measurements. In addition, Cohen (1983) now provides a better calibration of the abundances of the metal-rich clusters (based on observations of individual giants) than was available to Zinn (1980). We therefore use the abundances adopted from Cohen $(1982,1983)$ and her previous work, summarized in Table 4 , to calibrate $(V-K)_{0}(\mathrm{~GB})$ and $(J-K)_{0}(\mathrm{~GB})$ into metal abundances for the clusters.

Plots of the infrared colors of the cluster GB's against Cohen's $(1982,1983)$ metallicities are shown in Figures $12 a$ and $b$. In the calculation of the dependence of $[\mathrm{Fe} / \mathrm{H}]$ on the two colors we excluded from the $J-K$ solution those clusters with $[\mathrm{Fe} / \mathrm{H}] \geq-0.8$ because of the aforementioned decrease in sensitivity of the $J-K$ color of the giant branch to $T_{e}$ for cool temperatures, apparent in Figure 12b. The solutions are

$$
\begin{aligned}
{[\mathrm{Fe} / \mathrm{H}]_{\mathrm{Cohen}} } & =-6.905+6.329(J-K)_{0}(\mathrm{~GB}), \\
r & =0.90, \quad \sigma=0.16
\end{aligned}
$$

and

$$
\begin{aligned}
{[\mathrm{Fe} / \mathrm{H}]_{\mathrm{Cohen}} } & =-6.679+1.55(V-K)_{0}(\mathrm{~GB}), \\
r & =0.95, \quad \sigma=0.19 .
\end{aligned}
$$

\begin{tabular}{|c|c|c|}
\hline Cluster & {$[\mathrm{Fe} / \mathrm{H}]$} & Notes \\
\hline NGC 104 (47 Tuc) ... & -0.7 & 1 \\
\hline NGC 288 & -1.2 & 2 \\
\hline NGC 362 . & -1.25 & 2 \\
\hline NGC 3201 & -1.4 & 1 \\
\hline NGC $5272(\mathrm{M} 3) \ldots \ldots$ & -1.8 & 3 \\
\hline NGC 5904 (M5). & -1.45 & 1 \\
\hline NGC 5927. & -0.1 & 1 \\
\hline NGC 6171 & -0.9 & 1 \\
\hline NGC 6205 (M13) .... & -1.6 & 3 \\
\hline NGC 6341 (M92) .... & -2.35 & 4 \\
\hline NGC 6352 . & -0.3 & 1 \\
\hline NGC $6637 \ldots \ldots \ldots \ldots$ & -0.85 & 1 \\
\hline NGC $6656(\mathrm{M} 22) \ldots$ & -1.75 & 5 \\
\hline NGC $6838(M 71) \ldots$ & -0.75 & 1 \\
\hline NGC $7006 \ldots \ldots \ldots \ldots$ & -1.5 & 6 \\
\hline NGC 7078 (M15) .... & -2.2 & 4 \\
\hline
\end{tabular}

Equation $(7 b)$ is a marginally better fit to the data than
TABLE 4

Notes. - (1) From Cohen 1983. (2) From Cohen 1982 with an adjustment of +0.1 dex due to revision of 47 Tuc abundance in Cohen 1983. (3) From Cohen 1978. (4) From Cohen 1979. (5) From Cohen 1981. (6) From GC7.

is equation (1) for which the dispersion in $[\mathrm{Fe} / \mathrm{H}]_{\mathrm{Z}}$ is 0.24 .

Equations (7a) and (7b) were used to derive metallicities for each of the clusters in the present sample from the infrared colors. The mean difference in the metallicities derived from the two colors is 0.04 dex with a dispersion of 0.25 . The two values were averaged and the results tabulated in column (16) of Table 1 as $[\mathrm{Fe} / \mathrm{H}]_{\mathrm{IR}}$. Given the small dispersion between the fit of equations $(7 a)$ and $(7 b)$ to the spectroscopically determined $[\mathrm{Fe} / \mathrm{H}]$ values, the major source of uncertainty in the new scale is uncertainty in the reddening values used; namely,

$$
\Delta[\mathrm{Fe} / \mathrm{H}]_{\mathrm{IR}}=-3.7 \Delta[E(B-V)],
$$




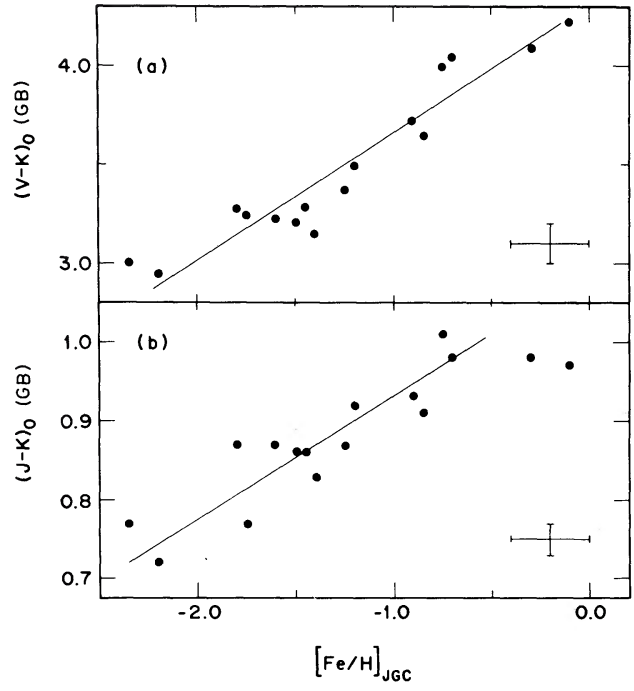

FIG. 12

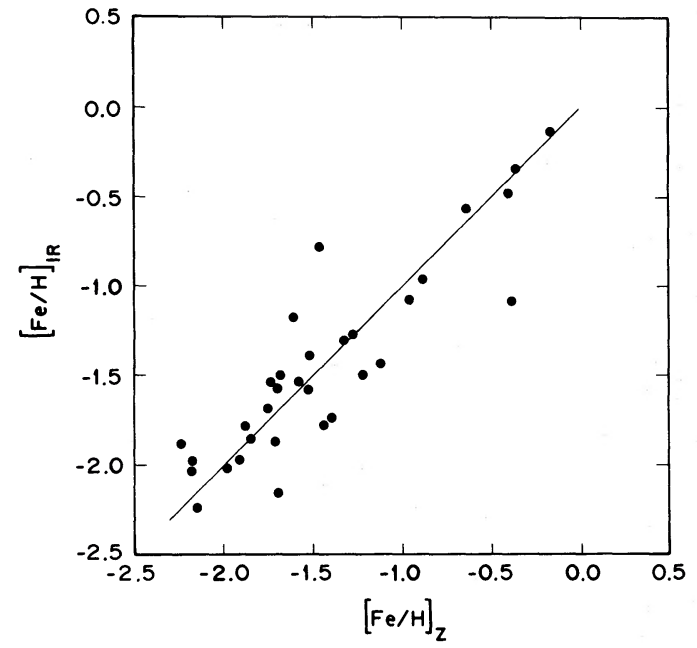

FIG. 13

Fig. 12. $-(V-K)_{0}(\mathrm{~GB})$ and $(J-K)_{0}(\mathrm{~GB})$ are from Table 1. $[\mathrm{Fe} / \mathrm{H}]$ values are from Cohen $(1982,1983)$ as given in Table 4 . Least squares fits to the data sets have been determined as in Fig. 2. The three clusters with $[\mathrm{Fe} / \mathrm{H}] \geq-0.8$ were not used in calculating the fit to $(J-K)_{0}(\mathrm{~GB})$ for reasons discussed in the text.

FIG. 13.-The two metallicity scales discussed in the paper are plotted against one another. The least squares fit is determined as in Fig. 2.

so an error of $0.04 \mathrm{mag}$ in $E(B-V)$ results in an error in $[\mathrm{Fe} / \mathrm{H}]_{\mathrm{IR}}$ greater than that due to uncertainties in the calibration.

How well does $[\mathrm{Fe} / \mathrm{H}]_{\mathrm{IR}}$ correlate with the other physical parameters discussed earlier? The dependence of $M_{K_{0}}(\mathrm{~GB})$ on $[\mathrm{Fe} / \mathrm{H}]_{\mathrm{IR}}$ is illustrated in Figure $4 b$, and is significantly tighter than its dependence on $[\mathrm{Fe} / \mathrm{H}]_{\mathrm{Z}}$ (shown in Fig. $4 a$ ). The least squares solution is

$$
M_{K_{0}}(\mathrm{~GB})=-2.99+1.192[\mathrm{Fe} / \mathrm{H}]_{\mathrm{IR}} \text {, }
$$

with $r=0.96$ and a dispersion of $0.18 \mathrm{mag}$. The good quality of the fit could be anticipated from Figure $4 a$ and the homologous nature of the cluster GBs. The mean effect of changes in $E(B-V)$ is

$$
\Delta\left[M_{K_{0}}(\mathrm{~GB})\right]=-5 \Delta[E(B-V)] .
$$

Therefore, reddening errors will move points parallel to the line in Figure $4 b$, not perpendicular to it.

It is informative to compare the IR metallicity scale and Zinn's directly (Fig. 13) and to understand the causes of any discrepancy. Clusters with problematical reddenings, distance moduli, or visual photometry have already been noted by the methods described in GC8 and in § III. Thus significant discrepancies between the Zinn scale and one based on the cluster GB parameters must be due to problems affecting the integrated light measurements in the blue only, as longward of $V$ we have shown in $\S \mathrm{V}$ that integrated light measurements are well predicted by GB parameters alone. The best fit least squares line is

$$
[\mathrm{Fe} / \mathrm{H}]_{\mathrm{IR}}=-0.07+0.95[\mathrm{Fe} / \mathrm{H}]_{\mathrm{Z}} \text {, }
$$

with $r=0.89$ and a dispersion, in $[\mathrm{Fe} / \mathrm{H}]_{\mathrm{IR}}$, of $0.24 \mathrm{dex}$. The dispersion is larger than would have been predicted from the internal errors, and we ask if this could be due to one of the problems which can affect integrated photometry of clusters. Manduca (1982) has calculated Zinn's (1980) metallicity indicator Q39 from spectra synthesized from stellar atmosphere codes and shown that because it is in the blue region of the spectrum, it can be significantly influenced by a cluster's HB morphology independent of the cluster's metallicity. This assertion can be tested by examining directly the dependence of the HB parameter on the two metallicity scales. This is done in Figures $8 a$ and $8 b$. Clearly, the HB parameter has a much poorer correlation with $[\mathrm{Fe} / \mathrm{H}]_{\mathrm{IR}}$ than with $[\mathrm{Fe} / \mathrm{H}]_{\mathrm{Z}}$. Aside from $\mathrm{M} 4$ which has problems that are discussed in $\S$ III $a$ and the Appendix, the main source of this difference arises from the shift by $0.2 \mathrm{dex}$ in the mean to relatively higher metallicities on the IR scale of the four circled clusters in Figure 8 which have exceptionally blue $\mathrm{HBs}$ for their metallicities on either scale-M10, M13, NGC 288, and NGC 6752 and NGC 5897 which was not in fact observed by Zinn (1980) and hence its $[\mathrm{Fe} / \mathrm{H}]_{Z}$ is quite uncertain. Thus, Manduca's claim that an exceptionlly blue $\mathrm{HB}$ will result in too low a metallicity being assigned on the basis of Zinn's (1980) Q39 values appears to be observationally confirmed. 
Finally we have compared the IR metallicity scale to the Searle and Zinn (1978) scale which is also based on observations of individual giant stars. However, rather than measuring individual metallic lines in the visual and red spectral region, they have determined a parameter $\langle S\rangle$ which is a measure of overall line blanketing in the spectral region between 3800 and $4800 \AA$. Agreement with the IR scale, based on Cohen's $(1982,1983)$ metallicities is reasonable, but we note the following curious fact: M3, M5, M10, M13, and NGC 7006 have a range of 0.5 dex in $[\mathrm{Fe} / \mathrm{H}]$ on Searle and Zinn's scale and yet the $(V-K)_{0}$ and $(J-K)_{0}$ colors of their giant branches are virtually identical (Table 1). This apparent difference in ranking for the five clusters is significantly greater than would be allowed by the internal photometric errors. The IR metallicity scale is also more tightly correlated with $(B-V)_{0, g}$ than is the Zinn scale, but that is only to be expected given the homologous nature of globular cluster giant branches as demonstrated in $\S$ III $a$.

\section{SUMMARY}

The extensive data base of infrared photometry of individual stars in 33 globular clusters (GC1-GC8) is used to determine characteristic parameters that describe each cluster as a whole. The globular cluster giant branches form a strictly homologous sequence in the observational (color-magnitude) and physical ( $T_{\text {eff }}$-luminosity) H-R diagrams. The deviations from the exceptionally tight mean relationships among the parameters describing the clusters' giant branches can be isolated to reddening errors or probable systematic errors in the visual photometry in particular individual cases. The ordering of cluster giant branches correlates well with the Zinn (1980) metallicity scale. The observed luminosities of the brightest giant in each cluster agree extremely well with the predicted location of the core helium flash as a function of metallicity. Furthermore, the very small dispersion in these luminosities at a given $[\mathrm{Fe} / \mathrm{H}]_{\mathrm{Z}}$, especially when the least luminous (sparsest) clusters are eliminated, confirms the widely held belief that the intrinsic scatter in the absolute magnitude of horizontalbranch stars of a given metallicity is small (less than 0.1 mag). The distribution of stars within $0.2 \mathrm{mag}$ of the red giant tip is consistent with that predicted from Monte
Carlo calculations given the range in cluster sparseness if the AGB extends all the way up the red giant branch to the tip.

The mean $\mathrm{CO}$ index increases as $[\mathrm{Fe} / \mathrm{H}]_{\mathrm{Z}}$ increases; the dispersion in this relationship is not larger than the relevant uncertainties, although a few cases can be found of clusters with similar metallicities but strong systematic differences in the $\mathrm{CO}$ strength (i.e., M3 and $\mathrm{M} 13)$. The $\mathrm{CO}$ residuals from the mean relationship of $\mathrm{CO}$ versus $[\mathrm{Fe} / \mathrm{H}]_{\mathrm{Z}}$ are not correlated with the $\mathrm{HB}$ residuals from the mean $\mathrm{HB}$ type as a function of metallicity. Thus the $\mathrm{CO}$ abundance is unrelated to the second parameter problem.

Within each metal rich cluster, the spread in $\mathrm{CO}$ appears to be comparable to that seen in 47 Tuc. The spread in the observed $\mathrm{CO}$ index is smaller in the metal-poor clusters and can be attributed solely to measuring uncertainties and statistical fluctuations, except for NGC 2808, 362, and M22. The latter two have spreads in CO which are as large as that seen in $\omega$ Cen.

The integrated light measurements of $(V-K)_{0}$ and $\mathrm{CO}$ by ACMM correlate well with the corresponding parameters derived here from the individual star data. The slope of these correlations is that expected based on standard models (such as those of ACMM) for the integrated light of old stellar populations. The integrated light of such systems, at least from $V$ to $K$, is now well understood.

To isolate any problems in the Zinn (1980) metallicity scale, the giant branch parameters are used to derive a metallicity ranking scheme, $[\mathrm{Fe} / \mathrm{H}]_{\mathrm{IR}}$. Clusters with exceptionally blue horizontal branches for their metallicity (on either abundance scale) are found to be ranked both relatively and absolutely with higher metallicities by this scheme than by $[\mathrm{Fe} / \mathrm{H}]_{\mathrm{Z}}$. This provides direct observational evidence that a systematic problem with $[\mathrm{Fe} / \mathrm{H}]_{\mathrm{Z}}$, based on blue integrated light spectral features, exists for clusters with unusually blue horizontal branches.

J. A. F. wishes to thank Marshall Cohen and the Caltech Astronomy Department for their hospitality while on sabbatical leave during 1982 when this paper was prepared. Particular thanks go to Jeff Pier for use of his plotting routine and to Mike Lesser for instruction in use of the VAX 11/780 computer.

\section{APPENDIX}

\section{DISCREPANT CLUSTERS}

Five clusters appear to be displaced from the mean $(J-K)_{0}(\mathrm{~GB})$ versus $[\mathrm{Fe} / \mathrm{H}]_{\mathrm{Z}}$ relation in Figure 3NGC 6553, 6637 (M69), 6121 (M4), 288, and 4372. These five are amongst the most deviant in Figures 2 and 4 as well. In the latter plot, NGC 2808 and 6397 are two other clusters which are significantly displaced from the rest. In the $M_{K_{0}}(\mathrm{~GB})$ versus $\log T_{e}(\mathrm{~GB})$ plot (Fig. 5) only M4 and NGC 2808 seem to be deviant. If we discount the possibility that our assumptions about the distance scale and the monotonic dependence of $\log T_{e}(\mathrm{~GB})$ on metallicity have exceptions, and if we stand by our claim for the high internal accuracy of the 
infrared data (GC8), what are the possible explanations for the discrepant points?

NGC 6553 lies in a very crowded field as is evident from the finding chart given by Hartwick (1975). A number of the giants in NGC 6553 have $(V-K)$ colors bluer by more than $0.5 \mathrm{mag}$ at constant $(J-K)$ than the narrow sequence defined by the other giants (Fig. $5 a$ of GC8). In a plot of $(J-K)_{0} \mathrm{~GB}$ against $\log T_{e}(\mathrm{~GB})$ only NGC 6553 is separated from the rest of the clusters. Also, given the cluster's high reddening, about 0.8 mag, and Hartwick's (1975) cautionary remarks about his photometry, its anomalous location in the various figures is not surprising. ${ }^{5}$

The infrared $C-M$ diagram for NGC 2808 is peculiar (GC8, Fig. 9). A $(J-K) C-M$ diagram is similar to one for NGC 1851 , whereas a $(V-K) C-M$ diagram is very different from that of NGC $1851-$ a cluster whose $[\mathrm{Fe} / \mathrm{H}]_{\mathrm{Z}}$ differs from NGC 2808 's by only 0.05 dex. This difference is evident in Figure $5 b$ of GC8: most of the NGC 2808 stars have $(V-K)$ 's too blue at a given $(J-K)$ by $0.2-0.3 \mathrm{mag}$. By looking at Figure 9 of GC8 one can verify that this will result in an $M_{K_{0}}(\mathrm{~GB})$ which is too bright by about $0.5 \mathrm{mag}$ and a $\log T_{e}(\mathrm{~GB})$ which is too hot. If there is a systematic error in the $V$ photometry so that the $(V-K)$ colors have to be changed by $0.2-0.3$, then NGC 2808 is put close to the line in Figures 2-4. ${ }^{6}$

There are three possible causes for the discrepant position of NGC 4372. First, its metallicity is not from Zinn (1980) and may be quite uncertain; $[\mathrm{Fe} / \mathrm{H}]_{\mathrm{IR}}$ is 0.4 dex less than that which we call $[\mathrm{Fe} / \mathrm{H}]_{\mathrm{Z}}$. Second, Figure $5 c$ in GC8 shows that $(V-K)_{0}$ for most of the stars is $0.2-0.3 \mathrm{mag}$ too red for their $(J-K)_{0}$ values. If we assume there is an error in the $V$ photometry, $M_{K_{0}}(\mathrm{~GB})$ will become brighter by about $0.4 \mathrm{mag}$, and the deduced $[\mathrm{Fe} / \mathrm{H}]_{\mathrm{IR}}$ would put NGC 4372 among the most metal-poor clusters. Then, it would no longer be discrepant in Figures 2 or 3 or 4 . Finally, its large reddening, $E(B-V)=0.45$ could be uncertain and spatially variable.

NGC 288 is noticeably separated from other clusters only in plots involving $[\mathrm{Fe} / \mathrm{H}]_{Z}$. As discussed in $\S \mathrm{VI}$, we believe that the exceptionally blue $\mathrm{HB}$ of this cluster

\footnotetext{
${ }^{5}$ Although not explicitly shown, the clusters NGC 6553, 2808, and 4372 are discrepant in a $M_{K_{0}}(\mathrm{~GB}),(J-K)_{0}(\mathrm{~GB})$ plot as well. The new values of $M_{K_{0}}(\mathrm{~GB})$ that would result from adjusting $(V-K)_{0}$ by the amounts that the clusters' stars are displaced from the mean $(V-K)_{0},(J-K)_{0}$ relation, would remove this discrepancy. [Recall that $M_{K_{0}}(\mathrm{~GB})$ is read at a fixed $(V-K)_{0}$ on the giant branch. Since the giant branches are quite steep, a shift in color of the GB will significantly alter the derived value of $M_{K_{0}}$.]

${ }^{6}$ Harris (1975) indicated that there are problems with Alcaino's (1971) photometry. We have already applied a correction to the latter's values (see Table 8 of GC8, but for the most part the stars we observed are too bright to be corrected using Harris's data. In fact, it seems from the discussion here that for these bright stars the correction has to go in the opposite sense from that which would be deduced from Harris's Fig. 3 (see also note 5).
}

has caused Zinn (1980) to underestimate its metallicity significantly. In addition, although the brighter and redder stars in NGC 288 form a tight sequence in $(J-K)_{0}$. $(V-K)_{0}$ (Fig. $5 c$ of GC8), they are systematically displaced about $0.1 \mathrm{mag}$ to the blue in $(V-K)$ of the mean cluster line. ${ }^{7}$ We conclude that $M_{K_{0}}(\mathrm{~GB})$ can be made a little fainter, $\log T_{e}(\mathrm{~GB})$, cooler, and $[\mathrm{Fe} / \mathrm{H}]$ larger than Zinn's value by $0.4-0.5$ dex. These changes would put NGC 288 close to the mean lines in Figures 2,3 , and 4 .

Now consider NGC 6121 (M4). The giant branch parameters for this cluster appear to be incorrect in the sense that the magnitudes are too faint (Figs. 4 and 5) and the colors too red, or the temperature too cool (Figs. 2 and 3, but note Fig. 5). As discussed in GC8, the overall appearance of the GB of this cluster points to an underestimation of the reddening, consistent with the conclusion of $\S \mathrm{III} a$ here. In addition, it is apparent from Figure $12 b$ of GC8 that the upper part of the GB of M4 is poorly defined because of the star-to-star scatter. If we increase $E(B-V)$ by $0.1 \mathrm{mag}$ and place our mean GB for M4 more to the blue of where we had originally placed it (it cannot be reasonably moved to the red, but a blueward shift is certaintly allowable), then we come up with the following values for the GB parameters of M4: $M_{K_{0}}(\mathrm{~GB})=-4.3, \log T_{e}(\mathrm{~GB})=$ 3.598, $(J-K)_{0}(\mathrm{~GB})=0.90$, and $(V-K)_{0}(\mathrm{~GB})=3.6$. These changes considerably reduce the discrepancies exhibited by M4 in Figures 2 through 5; a modest increase in $[\mathrm{Fe} / \mathrm{H}]_{\mathrm{Z}}$ for the cluster, would effectively eliminate them. The new value for $[\mathrm{Fe} / \mathrm{H}]_{\mathrm{IR}}$ is -1.1 , consistent with Cohen's (1983) abundance and with Cacciari's (1979) based on an RR Lyrae study, but somewhat higher than Zinn's (1980) value.

The relative location of M69 in Figures 2, 3 and 4 suggests that its reddening has been overestimated. Mould, Stutman, and McElroy (1979) have argued that $E(B-V)$ for $\mathrm{M} 69$ should be 0.1 rather than 0.17 . If this change is adopted, then the GB parameters become: $M_{K_{0}}(\mathrm{~GB})=-3.95, \quad(J-K)_{0}(\mathrm{~GB})=0.93, \quad(V-$ $K)_{0}(\mathrm{~GB})=3.70$, and $\log T_{e}(\mathrm{~GB})=3.584$. These changes reduce but do not remove the discrepancies in the various figures. Cohen's (1983; Table 4 here) spectroscopic criteria place M69 0.1 to 0.3 dex lower on the ranking ladder than Zinn's (1980) data, further helping matters. One can see from Figure 6 of GC8 that the

\footnotetext{
${ }^{7}$ There is no systematic difference between Cannon's (1974) and Alcaino's (1975) photometry for this cluster. Unfortunately, their two samples do not overlap in the $V, B-V$ region of interest here -i.e., that of the bright red stars which, with one exception, are all drawn from Alcaino's list. The six stars of Cannon's that we observed do not appear to depart significantly from the mean $J-K, V-K$ line. Since the bright red giants all lie in the central region of the cluster, a systematic error of $0.1 \mathrm{mag}$ in the correct sense could arise from a background contribution to the photographic photometry.
} 
infrared observations do not define the GB of M69 particularly well. If any of the stars are AGB stars, then the mean GB we used would have to be drawn further to the red. This would also cause changes in the GB parameters in the correct sense. Finally, we note that there is some uncertainty regarding the optical data (cf. Hartwick and Sandage 1968 and Harris 1977).

The discrepancies exhibited by NGC 6397 are relatively small compared to those of the clusters just discussed. As pointed out in $\mathrm{GC} 8$, the reddening value used may be off a bit, and there may be a problem involved with determining some of the GB parameters right at the tip of the GB. Almost any changes necessary to bring NGC 6397 closer to the mean lines in Figure 4 would be allowable by the available data and would be consistent with its locations in Figures 2 and 3.

From the semiquantitative analysis presented in this Appendix, we conclude that two major sources of scatter in diagrams which plot one GB parameter against another or against independently derived metallicity scales are systematic errors in the $V$ magnitudes and errors in the reddening values used. In fact, if such diagrams are truly dispersionless, then they, along with plots of $(V-K)_{0}$ against $(J-K)_{0}$ for individual stars, can be quite useful in selecting out clusters whose data suffer from errors of the types mentioned.

\section{REFERENCES}

Aaronson, M., Cohen, J. G., Mould, J., and Malkan, M. 1978, Ap. $J .$, 223, 823 (ACMM).

Alcaino, G. 1971, Astr. Ap., 15, 360. 1975, Astr. Ap. Suppl., 21, 15.

Baldwin, J. R., Frogel, J. A., and Persson, S. E. 1973, Ap. J., 184, 427.

Cacciari, C. 1979, A.J., 84, 1542

Cannon, R. D. 1974, M.N.R.A.S., 167, 551.

Christy, R. F. 1966, Ap. J., 144, 108.

Clube, S. V. M., and Dawe, J. A. 1978 in IAU Symposium 80, The HR Diagram ed. A. G. Davis Philip and D. S. Hayes (Dordrecht: Reidel), p. 53.

Cohen, J. G. 1978, Ap. J., 223, 487.

1979, Ap. J., 231, 751 .

1981, Ap.J., 247, 869

1982, Ap.J., 258, 143.

1983, Ap. J., 270, 654

Cohen, J.G., and Frogel, J. A. 1982, Ap. J. (Letters), 255, L39 $(\mathrm{GC} 7)$.

Cohen, J. G., Frogel, J. A., and Persson, S. E. 1978, Ap. J., 222, $165(\mathrm{GC} 1)$.

Cohen, J. G., Frogel, J. A., Persson, S. E., and Zinn, R. 1980, Ap. $J ., 239,74$ (GC4).

Da Costa, G. S., Frogel, J. A., and Cohen, J. G. 1981, Ap. J., 248, 612 (GC6).

Frogel, J. A. 1980, in IAU Symposium 85, Star Clusters, ed. J. E. Hesser (Dordrecht: Reidel), p. 435.

. 1983, Ap.J., 272, 167.

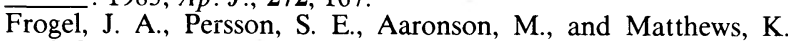
1978, Ap. J., 220, 75.

Frogel, J. A., Persson, S. E., and Cohen, J. G. 1979, Ap. J., 227,

$499(\mathrm{GC} 2)$.

1980, Ap. J., 240, 785.

—. $1981 a$, Ap. J., 246, 842 (GC5).

1981 b, in Physical Processes in Red Giants, ed. I. Iben, Jr.

Frogel, J. A., Persson, S. E., and Cohen, J. G. 1983, Ap. J. Suppl., 53, 713 (GC8).

Graham, J. A. 1973, in Variable Stars in Globular Clusters and Related Systems, ed. J. D. Fernie (Dordrecht: Reidel) p. 120. 1975, Pub. A.S.P., 87, 641

Harris, W. E. 1975, Ap. J. Suppl., 29, 397. 1977, Pub. A.S.P., 89, 482.

Harris, W. E., and Racine, R. 1979, Ann. Rev. Astr. Ap., 17, 241.

Hartwick, F. D. A. 1975, Pub. A.S.P., 87, 77.

Hartwick, F. D. A., and Sandage, A. 1968, Ap. J., 153, 715

Iben, I., Jr., and Truran, J. W. 1978, $A p . J ., 220,980$.

Kukarkin, B. V. 1974, The General Catalogue of Globular Star Clusters of Our Galaxy (Moscow: Publishing House Nauka).

Manduca, A. 1982, private communication.

Manduca, A., Bell, R. A., Barnes, T. G., Moffett, T. J., and Evans, D. S. 1981, Ap. J., 250, 312 .

Mould, J., Stutman, D., and McElroy, D. 1979, Ap. J., 228, 423.

Persson, S. E., Frogel, J. A., Cohen, J. G., Aaronson, M., and Matthews, K. 1980, Ap. J., 235, 452 (GC3).

Pilachowski, C. A. 1978, Ap. J., 224, 412.

Pilachowski, C. A., Sneden, C. and Green, E. 1981 in Astrophysical Parameters for Globular Clusters ed. A. G. Davis Philip and D. S. Hayes (Schenectady: L. Davis Press), p. 97.

Renzini, A. 1977, in Advanced Stages in Stellar Evolution, ed. P. Bouvier and A. Maeder (Geneva: Geneva Observatory), p. 149. Rood, R. T. 1972, Ap. J., 177, 681.

Sandage, A. 1982, Ap. J., 252, 553.

Searle, L., and Zinn, R. 1978, $A p . J ., 225,357$.

Sweigart, A. V., and Gross, P. G. 1978, Ap. J. Suppl., 36, 405.

van Albada, T. J., and Baker, N. 1973, Ap. J., 185, 477.

VandenBerg, D. A. 1983, Ap. J. Suppl., 51, 29.

Wood, P. R., and Cahn, J. H. 1977, Ap. J., 211, 499.

Zinn, R. 1980, Ap. J. Suppl., 42, 19.

and A. Renzini (Dordrecht: Reidel), p. 55.

Judith G. CoHEN: 105-24, California Institute of Technology, Pasadena, CA 91125

JAY A. Frogel: Cerro Tololo Inter-American Observatory, Casilla 601, La Serena, Chile

Sven ErIC Persson: Mount Wilson and Las Campanas Observatories, 813 Santa Barbara Street, Pasadena, CA 91101 